\title{
SELBERG ZETA FUNCTIONS ON ODD-DIMENSIONAL HYPERBOLIC MANIFOLDS OF FINITE VOLUME
}

\author{
JONATHAN PFAFF
}

\begin{abstract}
We study Selberg zeta functions $Z(s, \sigma)$ associated to locally homogeneous vector bundles over the unit-sphere bundle of a complete odd-dimensional hyperbolic manifold of finite volume. We assume a certain condition on the fundamental group of the manifold. A priori, the Selberg zeta functions are defined only for $s$ in some right halfspace of $\mathbb{C}$. We will prove that for any locally homogeneous bundle the functions $Z(s, \sigma)$ have a meromorphic continuation to $\mathbb{C}$ and we will give a complete description of their singularities in terms of spectral data of the underlying manifold. Our work generalizes results of Bunke and Olbrich to the non-compact situation. As an application of our results one can compare the normalized Reidemeister torsions on hyperbolic 3 manifolds with cusps which were introduced by Menal-Ferrer and Porti to the corresponding regularized analytic torsions.
\end{abstract}

\section{INTRODUCTION}

Let $X$ be a complete hyperbolic manifold of finite volume and of odd dimension $d$. Then it is possible to encode geometric data of $X$ associated to its geodesic flow into dynamical zeta functions, called Selberg zeta functions. These functions are associated to locally homogeneous vector bundles over the unit sphere bundle $S X$ of $X$. They are defined as an infinite product which converges only in some right half-space of the complex plane. The purpose of this paper is to prove a meromorphic continuation of the zeta functions associated to any locally homogeneous vector bundle to $\mathbb{C}$ and to relate their singularities to spectral data of $X$. In the non-compact case, such a result had previously been known only for a finite number of special bundles.

We shall now define the Selberg zeta functions more precisely. For further details we refer the reader to section 3. The tangent bundle $T S X$ of $S X$ is a locally homogeneous vector bundle and we fix an invariant metric. Let $\Phi$ be the geodesic flow of $X$ acting on $S X$. Let $d \Phi$ denote the differential of $\Phi$ with respect to $S X$. Then $\Phi$ has the Anosov property. This means that there is a $d \Phi$-invariant splitting

$$
T S X=T^{\mathrm{s}} S X \oplus T^{\mathrm{u}} S X \oplus T^{0} S X,
$$

where $d \Phi$ is exponentially shrinking on $T^{\mathrm{s}} S X$ and exponentially expanding on $T^{\mathrm{u}} S X$ as $t \rightarrow \infty$ with respect to the metric and where $T^{0} S X$ is the one-dimensional subspace tangent to the flow.

Denote by $\mathcal{C}(X)$ the set of non-trivial closed geodesics on $X$ parametrized by arc-length and for $c \in \mathcal{C}(X)$ let $\ell(c)$ be its length. The set $\mathcal{C}(X)$ corresponds bijectively to the closed orbits of $\Phi$. A closed geodesic $c \in \mathcal{C}(X)$ is called prime if it is the shortest among the 
closed geodesics having the same image as $c$. The set of prime geodesics will be denoted by $\mathcal{P C}(X)$. For $c \in \mathcal{C}(X)$ let $P_{c}:=\left.d \Phi\right|_{(\ell(c), \dot{c}(0))}$ be its monodromy map, also called the Poincaré map of $c$. Then $P_{c}$ respects the splitting (1.1) and its restriction to the fibre of $T^{\mathrm{s}} S X$ over $\dot{c}(0)$ will be denoted by $P_{c}^{\mathrm{s}}$.

Now we recall that $X$ can be realized as $X=\Gamma \backslash \tilde{X}$, where $\tilde{X}=G / K$ with $G=\operatorname{Spin}(d, 1)$, $K=\operatorname{Spin}(d)$ and where $\Gamma$ is a discrete, torsion-free subgroup of $G$. The space $\widetilde{X}$ can be identified with the $d$-dimensional hyperbolic space. Let $P_{0}:=M A N$ be the standard parabolic subgroup of $G$ and let $\sigma$ be a finite dimensional unitary representation of $M$. Then $\sigma$ naturally defines a locally homogeneous vector bundle $V(\sigma)$ over $S X$ and the geodesic flow lifts to a flow on $V(\sigma)$. Thus for every closed geodesic $c$ its lift to $V(\sigma)$ defines an endomorphism $\mu_{\sigma}(c)$ on the fibre of $V(\sigma)$ over $\dot{c}(0)$. Let $d=2 n+1$, then the Selberg zeta function $Z(s, \sigma)$ is defined as

$$
Z(s, \sigma):=\prod_{c \in \mathcal{P} \mathcal{C}(X)} \prod_{k=0}^{\infty} \operatorname{det}\left(\operatorname{Id}-\mu_{\sigma}(c) \otimes S^{k} P_{c}^{\mathrm{s}} e^{-(s+n) \ell(c)}\right) .
$$

Here $S^{k}$ denotes the $k$-th symmetric power of an endomorphism. The infinite product in (1.2) converges absolutely only for $\operatorname{Re}(s)>2 n$. In this paper we prove that $Z(s, \sigma)$ has a meromorphic continuation to $\mathbb{C}$ and describe its singularities.

From now on we assume that $\Gamma$ satisifies the following condition: For every $\Gamma$-cuspidal parabolic subgroup $P=M_{P} A_{P} N_{P}$ of $G$ one has

$$
\Gamma \cap P=\Gamma \cap N_{P} .
$$

The central elements of our main result can be summarized as follows.

Theorem 1.1. Let $X$ be a complete odd dimensional hyperbolic manifold of finite volume and assume that its fundamental group $\Gamma$ satisfies (1.3). Let $\sigma$ be a finite dimensional unitary representation of $M$. Then the Selberg zeta function $Z(s, \sigma)$ has a meromorphic continuation to $\mathbb{C}$. All its possible singularities (zeroes and poles) and their corresponding orders can be described in terms of the following data:

- by the discrete spectrum of a graded differential operator $A(\sigma)$ of Laplace type and of a twisted Dirac operator $D(\sigma)$ which act on a locally homogeneous vector bundle $E(\sigma)$ over $X$.

- by the poles of the scattering matrix $\mathbf{C}\left(\nu_{\sigma}: \sigma: s\right)$ associated to $\sigma$ and a certain representation $\nu_{\sigma}$ of $K$.

Additionally, the Selberg zeta function has singularities which are independent of $X$ and whose order depends only on $p$, the number of cusps of $X$.

Theorem 1.1 provides a relation between the geometry of the possibly non-compact hyperbolic manifold $X$ and the spectrum of certain differential operators. More precisely, the singularities in the first two items correspond to spectral parameters of $X$ since poles of the scattering matrix $\mathbf{C}\left(\nu_{\sigma}: \sigma: s\right)$ are related to poles of the resolvent of $A(\sigma)$.

The additional singularities of $Z(s, \sigma)$ on the negative real line arise from the contribution of weighted orbital integrals to the geometric side of the trace formula. For a more 
precise version of Theorem 1.1 we refer the reader to Theorem 9.2 at the end of this article .

Our main result implies the existence of the analytic continuation of the Ruelle zeta functions associated to representations of $M$ and $G$. These functions are dynamical zeta functions, defined as follows. Let $\sigma$ be a finite dimensional unitary representation of $M$. Then put

$$
R(s, \sigma):=\prod_{c \in \mathcal{P} \mathcal{C}(X)} \operatorname{det}\left(\operatorname{Id}-\mu_{\sigma}(c) e^{-s \ell(c)}\right) .
$$

Similarly, if $\tau$ is a finite dimensional representation of $G$, the restriction of $\tau$ to $\Gamma$ defines a locally homogeneous vector bundle $V(\tau)$ over $S X$ and the geodesic flow lifts to a flow on $V(\tau)$. Thus every closed geodesic $c$ induces an endomorphism $\mu_{\tau}(c)$ on the fibre of $V(\tau)$ over $\dot{c}(0)$. Now we define the Ruelle zeta function on $X$ associated to $\tau$ by

$$
R(s, \tau):=\prod_{c \in \mathcal{P} \mathcal{C}(X)} \operatorname{det}\left(\operatorname{Id}-\mu_{\tau}(c) e^{-s \ell(c)}\right) .
$$

The infinite products in (1.4) and (1.5) converge absolutely and locally uniformly only for $s \in \mathbb{C}$ with $\operatorname{Re}(s)$ sufficiently large. However one can express each Ruelle zeta function as a weighted product of Selberg zeta functions. Thus Theorem 1.1 implies the following corollary.

Corollary 1.2. For every finite dimensional unitary representation $\sigma$ of $M$ and for every finite dimensional irreducible representation $\tau$ of $G$ the Ruelle zeta functions $R(s, \sigma)$ and $R(s, \tau)$ admit a meromorphic continuation to $\mathbb{C}$.

We shall now describe an application of our methods and our main results to the 3dimensional case. In this case there is a natural isomorphism $G \cong \mathrm{SL}_{2}(\mathbb{C})$. For $m \in \frac{1}{2} \mathbb{N}$ let $\tau(m)$ be the $2 m$-th symmetric power of the standard representation of $\mathrm{SL}_{2}(\mathbb{C})$. Then in [MP2 we introduced the analytic torsion $T_{X}(\tau(m))$ of $X$ with coefficients in the locally homogeneous vector bundle defined by the restriction of $\tau(m)$ to $\Gamma$. This definition extends the definition of the analytic torsion on closed manifolds with coefficients in a local system to a specific non-compact situation. Now, recalling the Cheeger-Müller theorems, [Ch],

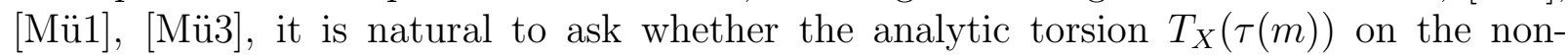
compact manifold $X$ has a combinatorial analogue.

In a subsequent paper, we will give a partial answer to this question by combining the methods and results of this paper with the recent work MePo of Menal-Ferrer and Porti on Reidemeister torsion and Ruelle zeta functions. To state our result, let $\bar{X}$ be the Borel-Serre compactification of $X$. Then $\bar{X}$ is a compact smooth manifold with boundary which is homotopy-equivalent to $X$ and $X$ is diffeomorphic to the interior of $\bar{X}$. Let $\bar{E}_{\tau(m)}$ denote the flat vector-bundle over $\bar{X}$ associated to the restriction of $\tau(m)$ to $\Gamma$, the fundamental group of $\bar{X}$. Then Menal-Ferrer and Porti introduced a canoncial family $\left\{\theta_{i}\right\}$ of bases of the singular homology groups of $\bar{X}$ with coefficients in $\bar{E}_{\tau(m)} \mathbb{M e P O}$, Proposition 2.10]. Moreover, they showed that for each $m \in \mathbb{N}, m \geq 3$, the quotient of the corresponding Reidemeister torsions $\frac{\left|\tau_{X}\left(\tau(m) ;\left\{\theta_{i}\right\}\right)\right|}{\left|\tau_{X}\left(\tau(2) ;\left\{\theta_{i}\right\}\right)\right|}$ is independent of the choice of the 
family $\left\{\theta_{i}\right\} \mathbb{M e P o}$, Proposition 2.2]. It is also independent of a given spin structure. We shall denote this quotient simply by $\frac{\mid \tau_{X}(\tau(m) \mid}{\left|\tau_{X}(\tau(2))\right|}$ here. We remark that our parametrization of the representations $\tau(m)$ is different from that used in $\mathrm{MePO}$ but consistent with [MP1], [MP2]. We will prove the following theorem.

Theorem 1.3. Let $X$ be a complete hyperbolic 3-manifold of finite volume and assume that its fundamental group $\Gamma$ satisfies (1.3). Then for each $m \in \mathbb{N}$, there exists an explicit constant $c(m)$, which is independent of $X$, such that for $m \geq 3$ one has

$$
\frac{T_{X}(\tau(m))}{T_{X}(\tau(2))}=\left(\frac{c(m)}{c(2)}\right)^{p} \frac{\left|\tau_{X}(\tau(m))\right|}{\left|\tau_{X}(\tau(2))\right|},
$$

where $p$ denotes the number of cusps of $X$. For $m \in \frac{1}{2} \mathbb{N}$, a similar formula holds.

If one applies the results of [MP2] and the explicit form of the constants $c(m)$, it follows that the quotients of torsions on both side of the equation are by no means identically one and are even exponentially growing as $m \rightarrow \infty$.

The proof of Theorem 1.3 will be given in another publication. It is based on relating the analytic torsion $T_{X}(\tau(m))$ to the behaviour of the Ruelle zeta function $R(s, \tau(m))$ at 0 . To prove this relation, we will express the Ruelle zeta function $R(s, \tau(m))$ by twisted Selberg zeta functions and then we will apply the methods and results of this paper. In particular, we will deduce a functional equation and a determinant formula for the Selberg zeta functions.

One can apply Theorem 1.3 to study for fixed $m \in \mathbb{N}$ the growth of the torsion in the cohomology $H^{*}\left(\Gamma_{i}, M_{\tau(m)}\right)$ for special sequences of arithmetic groups $\Gamma_{i}, i \in \mathbb{N}$. Here $M_{\tau(m)}$ denotes a lattice in the representation space $V_{\tau(m)}$ of $\tau(m)$ which is stable under the $\Gamma_{i}$. This gives a modified extension of some results of Bergeron and Venkatesh [BV] to the case of non-compact hyperbolic 3-manifolds of finite volume.

Let us now recall some of the previous results related to Theorem 1.1. If $X$ is compact, it was shown in $[\mathrm{Fr}]$ that $Z(s, \sigma)$ has a meromorphic continuation to $\mathbb{C}$. In a next step, combining the trace formula with spectral-theoretic methods, for compact X Bunke and Olbrich described the singularities of $Z(s, \sigma)$ as in the first item of Theorem [1.1 [BO]. If $X$ is only of finite volume and satisfies assumption (1.3), the meromorphic continuation of the Selberg zeta function and a description of its singularities has previously been obtained only for a finite number of special representations of $M$. Namely Gangolli and Wallach GaWa solved this problem for the trivial representation of $M$ and Gon and Park [GP] generalized their methods to treat the fundamental representations $\sigma_{k}, k=1, \ldots, n$ of $M$ on $\Lambda^{k}\left(\mathbb{C}^{2 n}\right)$. However, it is not clear whether the methods of Gangolli, Wallach, Gon and Park can be applied to general $\sigma \in \hat{M}$ since they use a special type of a Paley-Wiener theorem for differential forms which prescribes the $K$-types of a test function in a very specific way. This theorem exists only for the fundamental representation $\sigma_{k}, k=1, \ldots, n$. However, for an application as our proof of Theorem 1.3, it is necessary to understand the Selberg zeta function for every representation $\sigma$ of $M$. 
To prove our main result, we will generalize the approach of Bunke and Olbrich [BO] from the compact case to our situation. In the approach of Bunke and Olbrich to the Selberg zeta functions, the generalized Laplace operators appear quite naturally. To carry over their methods to the non-compact situation we use the invariant trace formula as it is stated and proved by Hoffmann [Ho2]. We would like to emphasize that the invariant trace formula can be applied only because the Fourier transform of the invariant part associated to the weighted orbital integral has been computed explicitly by Hoffmann [Ho1]. Our approach to the Selberg zeta function is different from that used by Gon and Park, since the trace formula they use is not invariant and so they have to prescribe the $K$-types of their test functions.

We shall now describe our proof more precisely. Let us first recall the setting of the trace formula. The right regular representation $\pi_{\Gamma}$ of $G$ on $L^{2}(\Gamma \backslash G)$ splits as $\pi_{\Gamma}=\pi_{\Gamma, d} \oplus \pi_{\Gamma, c}$. Here the representation $\pi_{\Gamma, d}$ is completely reducible. On the other hand, the representation $\pi_{\Gamma, c}$ is isomorphic to a direct integral over all tempered principle series representations of $G$. For a K-finite Schwarz function $\phi$ on $G$, the operator $\pi_{\Gamma, d}(\phi)$ is trace class and the invariant trace formula as it is stated in Ho2 expresses $\operatorname{Tr}\left(\pi_{\Gamma, d}(\phi)\right)$ as a sum of invariant distributions on $G$ applied to $\phi$.

In order to prove the meromorphic continuation of the Selberg zeta function $Z(s, \sigma)$, we first study the symmetrized Selberg zeta function $S(s, \sigma)$, which is given by $Z(s, \sigma)$ if $\sigma=w_{0} \sigma$ and by $Z(s, \sigma) Z\left(s, w_{0} \sigma\right)$, if $\sigma \neq w_{0} \sigma$. Here $w_{0}$ is a fixed representative of the restricted Weyl group. As in the compact case [BO], there is a $K$-finite function $h_{t}^{\sigma}$, belonging to all Harish-Chandra Schwarz spaces, such that the logarithmic derivative of $S(s, \sigma)$ is equal to a certain integral transform of $H\left(h_{t}^{\sigma}\right)$. Here $H$ is a distribution on $G$ which occurs in the invariant Selberg trace formula. It is built from the semisimple conjugacy classes of $\Gamma$. Geometrically, the function $h_{t}^{\sigma}$ arises from the graded fibre trace of the kernel of $e^{-t \tilde{A}(\sigma)}$, where $\tilde{A}(\sigma)$ is a Laplace-type operator which acts on a graded vector bundle $\tilde{E}(\sigma)$ over $\widetilde{X}$. Now we apply the invariant trace formula to $h_{t}^{\sigma}$. We study the integral transform of all involved summands carefully and in this way we obtain an expression for the logarithmic derivative of $S(s, \sigma)$.

If $\sigma$ is not invariant under the Weyl group, we introduce the antisymmetric Selberg zeta function $S_{a}(s, \sigma):=Z(s, \sigma) / Z\left(s, w_{0} \sigma\right)$. The bundle $\tilde{E}(\sigma)$ turns out to be a spinor bundle and there is a canonical twisted Dirac operator $\tilde{D}(\sigma)$ on $\tilde{E}(\sigma)$ such that $\tilde{D}(\sigma)^{2}=\tilde{A}(\sigma)$. Now the fibre trace of the kernel of $\operatorname{Tr}\left(\tilde{D}(\sigma) e^{-t \tilde{D}(\sigma)^{2}}\right)$ is represented by a $K$-finite HarishChandra Schwarz function $k_{t}^{\sigma}$ and the logarithmic derivative of $S_{a}(s, \sigma)$ equals an integral transform of $H\left(k_{t}^{\sigma}\right)$, where the distribution $H$ is as above. Using the invariant trace formula again we can study the logarithmic derivative of $S_{a}(s, \sigma)$. Putting everything together, we can complete the proof of Theorem 1.1.

This paper is organized as follows. In section 2 we fix some notation and recall some basic facts concerning the group $G$. In section 3 we describe the Selberg zeta functions in terms which are needed for our approach via the trace formula. In section 4 we review Hoffman's 
factorization of the $C$-matrix associated to the Eisenstein series which is an important tool to state the invariant trace formula. The latter formula is treated in section 5. In the same section we study the Fourier transform of a certain distribution $\mathcal{I}$ occuring in the trace formula. In section 6 we introduce certain Laplace operators which act on graded locally homogeneous vector bundles over $X$ and compute the Fourier transform of the corresponding heat kernels. The meromorphic continuation of the symmetric Selberg zeta function and a description of its singularities is treated in section 7 . Section 8 is devoted to the study of certain twisted Dirac operators on locally homogeneous bundles over $X$. In the final section 9 we study the antisymmetric Selberg zeta function and complete the proof of Theorem 1.1.

Acknowledgement. This paper contains parts of the author's PhD thesis. He would like to thank his supervisor Prof. Werner Müller for his constant support and for helpful suggestions.

\section{Preliminaries}

In this section we establish some notation and recall some basic facts about representations of the involved Lie groups.

2.1. For $d \in \mathbb{N}, d=2 n+1$ we let $G:=\operatorname{Spin}(d, 1)$. The group $G$ is defined as the universal covering group of $\mathrm{SO}_{0}(d, 1)$, where $\mathrm{SO}_{0}(d, 1)$ is the identity component of $\mathrm{SO}(d, 1)$. Let $K:=\operatorname{Spin}(d)$. Then $K$ is a maximal compact subgroup of $G$. Put $\widetilde{X}:=G / K$. Let $G=N A K$ be the standard Iwasawa decomposition of $G$ and let $M$ be the centralizer of $A$ in $K$. Then we have $M=\operatorname{Spin}(d-1)$. The Lie algebras of $G, K, A, M$ and $N$ will be denoted by $\mathfrak{g}, \mathfrak{k}, \mathfrak{a}, \mathfrak{m}$ and $\mathfrak{n}$, respectively. Define the standard Cartan involution $\theta: \mathfrak{g} \rightarrow \mathfrak{g}$ by $\theta(Y):=-Y^{t}, \quad Y \in \mathfrak{g}$. Let $\mathfrak{g}:=\mathfrak{k} \oplus \mathfrak{p}$ be the Cartan decomposition of $\mathfrak{g}$ with respect to $\theta$. Let $B$ be the Killing form of $\mathfrak{g}$. Then $B$ is positive on $\mathfrak{p}$. We define a symmetric bilinear form $\langle\cdot, \cdot\rangle$ on $\mathfrak{g}$ by $\left\langle Y_{1}, Y_{2}\right\rangle:=\frac{1}{2(d-1)} B\left(Y_{1}, Y_{2}\right), Y_{1}, Y_{2} \in \mathfrak{g}$. Let $x_{0}=e K \in \widetilde{X}$. Then we have a canonical isomorphism $T_{x_{0}} \widetilde{X} \cong \mathfrak{p}$ and thus the restriction of $\langle\cdot, \cdot\rangle$ to $\mathfrak{p}$ defines an inner product on $T_{x_{0}} \widetilde{X}$ and therefore an invariant metric on $\widetilde{X}$. This metric has constant curvature -1 and $\widetilde{X}$, equipped with this metric, is isometric to the hyperbolic space $\mathbb{H}^{d}$.

2.2. Let $\mathfrak{a}$ be the Lie-Algebra of $A$. Then $\operatorname{dim}(\mathfrak{a})=1$. Fix a Cartan-subalgebra $\mathfrak{b}$ of $\mathfrak{m}$. Then $\mathfrak{b}$ is also a Cartan subalgebra of $\mathfrak{k}$ and $\mathfrak{h}:=\mathfrak{a} \oplus \mathfrak{b}$ is a Cartan-subalgebra of $\mathfrak{g}$. We can identify $\mathfrak{g}_{\mathbb{C}} \cong \mathfrak{s o}(d+1, \mathbb{C})$. Let $e_{1} \in \mathfrak{a}^{*}$ be the positive restricted root defining $\mathfrak{n}$ and let $H_{1} \in \mathfrak{a}$ such that $e_{1}\left(H_{1}\right)=1$. We fix $e_{2}, \ldots, e_{n+1} \in i \mathfrak{b}^{*}$ such that the positive roots $\Delta^{+}\left(\mathfrak{g}_{\mathbb{C}}, \mathfrak{h}_{\mathbb{C}}\right)$ are chosen as in $G W$, page 102] for the root system $D$. We let $\Delta^{+}\left(\mathfrak{g}_{\mathbb{C}}, \mathfrak{a}_{\mathbb{C}}\right)$ be the set of roots of $\Delta^{+}\left(\mathfrak{g}_{\mathbb{C}}, \mathfrak{h}_{\mathbb{C}}\right)$ which do not vanish on $\mathfrak{a}_{\mathbb{C}}$. The positive roots $\Delta^{+}\left(\mathfrak{m}_{\mathbb{C}}, \mathfrak{b}_{\mathbb{C}}\right)$ are chosen such that they are restrictions of elements from $\Delta^{+}\left(\mathfrak{g}_{\mathbb{C}}, \mathfrak{h}_{\mathbb{C}}\right)$. For $\alpha \in \Delta^{+}\left(\mathfrak{g}_{\mathbb{C}}, \mathfrak{h}_{\mathbb{C}}\right)$ there exists a unique $H_{\alpha}^{\prime} \in \mathfrak{h}_{\mathbb{C}}$ such that $B\left(H, H_{\alpha}^{\prime}\right)=\alpha(H)$ for all $H \in \mathfrak{h}_{\mathbb{C}}$. One has $\alpha\left(H_{\alpha}^{\prime}\right) \neq 0$. We let $H_{\alpha}:=\frac{2}{\alpha\left(H_{\alpha}^{\prime}\right)} H_{\alpha}^{\prime}$. For $j=1, \ldots, n+1$ we let $\rho_{j}:=n+1-j$. Then 
the half-sum of positive roots $\rho_{G}$ and $\rho_{M}$ of $\Delta^{+}\left(\mathfrak{g}_{\mathbb{C}}, \mathfrak{h}_{\mathbb{C}}\right)$ resp. $\Delta^{+}\left(\mathfrak{m}_{\mathbb{C}}, \mathfrak{b}_{\mathbb{C}}\right)$ are given by $\rho_{G}=\sum_{j=1}^{n+1} \rho_{j} e_{j} \operatorname{resp} \rho_{M}=\sum_{j=2}^{n+1} \rho_{j} e_{j}$.

2.3. Let $\mathbb{Z}\left[\frac{1}{2}\right]^{j}$ be the set of all $\left(k_{1}, \ldots, k_{j}\right) \in \mathbb{Q}^{j}$ such that all $k_{i}$ are integers or all $k_{i}$ are half integers. Then the finite dimensional representations $\nu \in \hat{K}$ of $K$ are parametrized by their highest weights $\Lambda \nu \in i \mathfrak{b}^{*}, \Lambda(\nu)=k_{2}(\nu) e_{2}+\cdots+k_{n+1}(\nu) e_{n+1},\left(k_{2}(\nu), \ldots, k_{n+1}(\nu)\right) \in$ $\mathbb{Z}\left[\frac{1}{2}\right]^{n}, k_{2}(\nu) \geq k_{3}(\nu) \geq \ldots \geq k_{n+1}(\nu) \geq 0$. The finite dimensional irreducible representations $\sigma \in \hat{M}$ of $M$ are parametrized by their highest weights $\Lambda(\sigma) \in i \mathfrak{b}^{*}$,

$$
\Lambda(\sigma)=k_{2}(\sigma) e_{2}+\cdots+k_{n+1}(\sigma) e_{n+1} ; \quad k_{2}(\sigma) \geq k_{3}(\sigma) \geq \cdots \geq k_{n}(\sigma) \geq\left|k_{n+1}(\sigma)\right|,
$$

where $\left(k_{2}(\sigma), \ldots, k_{n+1}(\sigma)\right) \in \mathbb{Z}\left[\frac{1}{2}\right]^{n}$. Let $M^{\prime}$ be the normalizer of $A$ in $K$ and let $W(A)=$ $M^{\prime} / M$ be the restricted Weyl-group. It has order two. Let $w_{0} \in W(A)$ be the non-trivial element. Then for $\sigma \in \hat{M}$ there is an associated representation $w_{0} \sigma$, see [MP2, section 2.4]. If the highest weight of $\sigma$ is as in (2.1), then the highest weight $\Lambda\left(w_{0} \sigma\right)$ of $w_{0} \sigma$ is given by $\Lambda\left(w_{0} \sigma\right)=k_{2}(\sigma) e_{2}+\cdots+k_{n}(\sigma) e_{n}-k_{n+1}(\sigma) e_{n+1}$. For $\nu \in \hat{K}$ and $\sigma \in \hat{M}$ we denote by $[\nu: \sigma]$ the multiplicity of $\sigma$ in the restriction of $\nu$ to $M$.

2.4. Let $\kappa$ be the spin-representation of $K$ over the spinor space $\Delta^{2 n}$. Then $\kappa$ is the representation with highest weight $\Lambda(\kappa)=\frac{1}{2} e_{2}+\cdots+\frac{1}{2} e_{n+1}$. By [GW, Theorem 8.1.4] there is an $M$-invariant splitting $\Delta^{2 n}=\Delta_{+}^{2 n} \oplus \Delta_{-}^{2 n}$ such that the restriction of $\kappa$ to $M$ acts on $\Delta_{+}^{2 n}$ as $\kappa^{+}$and on $\Delta_{-}^{2 n}$ as $\kappa^{-}$, where $\kappa^{ \pm}$are the representation of $M$ with highest weights $\frac{1}{2} e_{2}+\cdots+\frac{1}{2} e_{n} \pm \frac{1}{2} e_{n+1}$. Let $R(K)$ and $R(M)$ be the representation rings of $K$ and $M$. Let $\iota: M \longrightarrow K$ be the inclusion and let $\iota^{*}: R(K) \longrightarrow R(M)$ be the induced map. If $R(M)^{W(A)}$ is the subring of $W(A)$-invariant elements of $R(M)$, then clearly $\iota^{*}$ maps $R(K)$ into $R(M)^{W(A)}$. Moreover, the following proposition holds.

Proposition 2.1. The map $\iota$ is an isomorphism from $R(K)$ onto $R(M)^{W(A)}$. Let $\sigma \in \hat{M}$ be of highest weight $\Lambda(\sigma)$ as in (2.1) and assume that $k_{n+1}(\sigma)>0$. Let $\nu(\sigma) \in \hat{K}$ be the representation of highest weight $\Lambda(\nu(\sigma)):=\sum_{j=2}^{n+1}\left(k_{j}(\sigma)-1 / 2\right) e_{j}$. Then one has $\sigma-w_{0} \sigma=\left(\kappa^{+}-\kappa^{-}\right) \otimes \iota^{*} \nu(\sigma)$. Moreover, $\nu(\sigma) \otimes \kappa$ splits as $\nu(\sigma) \otimes \kappa=\nu^{+}(\sigma) \oplus \nu^{-}(\sigma)$ such that $\sigma+w_{0} \sigma=\iota^{*} \nu^{+}(\sigma)-\iota^{*} \nu^{-}(\sigma)$.

Proof. This is proved by Bunke and Olbrich, [BO], Proposition 1.1.

2.5. We parametrize the principal series as follows. Given $\sigma \in \hat{M}$ with $\left(\sigma, V_{\sigma}\right) \in \sigma$, let $\mathcal{H}^{\sigma}$ denote the space of $V_{\sigma}$-valued $L^{2}$-functions on $K$ which satisfy $\Phi(m k)=\sigma(m) \Phi(k)$ for allmost all $k \in K$, all $m \in M$. Then for $\lambda \in \mathbb{C}$ and $\Phi \in H^{\sigma}$ we define $\pi_{\sigma, \lambda}(g) \Phi(k):=$ $e^{(i \lambda+n) H(k g)} \Phi(\kappa(k g))$. Recall that the representations $\pi_{\sigma, \lambda}$ are unitary iff $\lambda \in \mathbb{R}$. Moreover, by $\left[\mathbb{K n}\right.$, Theorem 7.2, Theorem 7.12], $\pi_{\sigma, \lambda}$ and $\pi_{\sigma^{\prime}, \lambda^{\prime}}, \lambda, \lambda^{\prime} \in \mathbb{C}$ are equivalent iff either $\sigma=\sigma^{\prime}, \lambda=\lambda^{\prime}$ or $\sigma^{\prime}=w_{0} \sigma, \lambda^{\prime}=-\lambda$. Let $\nu \in \hat{K}$. The restriction of $\pi_{\sigma, \lambda}$ to $K$ coincides with the induced representation $\operatorname{Ind}_{M}^{K}(\sigma)$ and thus by Frobenius reciprocity $[\overline{K n}$, p.208] the multiplicity of $\nu$ in $\pi_{\sigma, \lambda}$ equals $[\nu: \sigma]$. By [GW, Theorem 8.1.4] one has $[\nu: \sigma] \leq 1$. Let $c(\sigma):=\sum_{j=2}^{n+1}\left(k_{j}(\sigma)+\rho_{j}\right)^{2}-\sum_{j=1}^{n+1} \rho_{j}^{2}$. Then, if $\Omega$ is the Casimir element with respect to 
the normalized Killing form one has $\pi_{\sigma, \lambda}(\Omega)=-\lambda^{2}+c(\sigma)$, see [MP1, Corollary 2.4]. By $\Theta_{\sigma, \lambda}$ we will denote the global character of $\pi_{\sigma, \lambda}$.

2.6. We let $\Gamma$ be a discrete, torsion free subgroup of $G$ with $\operatorname{vol}(\Gamma \backslash G)<\infty$ and we assume that $\Gamma$ satisfies (1.3) We let

$$
X:=\Gamma \backslash G / K
$$

We equip $X$ with the Riemannian metric induced from $\tilde{X}$. Let $\mathfrak{P}$ be a fixed set of representatives of $\Gamma$-inequivalent cuspidal parabolic subgroups of $G$. Then $\mathfrak{P}$ is finite. Let $p:=\# \mathfrak{P}$. Then $p$ equals the number of cusps of $X$. Let $P_{0}:=M A N$. Without loss of generality we will assume that $P_{0} \in \mathfrak{P}$. For every $P \in \mathfrak{P}$, there exists a $k_{P} \in K$ such that $P=N_{P} A_{P} M_{P}$ with $N_{P}=k_{P} N k_{P}^{-1}, A_{P}=k_{P} A k_{P}^{-1}, M_{P}=k_{P} M k_{P}^{-1}$. We let $k_{P_{0}}=1$. If $P \in \mathfrak{P}, P^{\prime} \in \mathfrak{P}$ we will say that $\sigma_{P} \in \hat{M}_{P}$ and $\sigma_{P^{\prime}} \in \hat{M}_{P^{\prime}}$ are associated if for all $m_{P^{\prime}} \in M_{P^{\prime}}$ one has $\sigma_{P^{\prime}}\left(m_{P^{\prime}}\right)=\sigma_{P}\left(k_{P} k_{P^{\prime}}^{-1} m_{P^{\prime}} k_{P^{\prime}} k_{P}^{-1}\right)$. For $\sigma_{P} \in \hat{M}_{P}$ we will denote by $\boldsymbol{\sigma}$ the set of all $\sigma_{P^{\prime}} \in \hat{M}_{P^{\prime}}$ associated to $\sigma_{P}$, where $P^{\prime}$ runs through $\mathfrak{P}$. For $g \in G$, we define $n_{P}(g) \in N_{P}$, $H_{P}(g) \in \mathbb{R}$ and $\kappa_{P}(g) \in K$ by $g=n_{P}(g) \exp \left(H_{P}(g) H_{1}\right) \kappa_{P}(g)$. We let $H(g):=H_{P_{0}}(g)$.

\section{Selberg ZETA FUnCtions}

This section is devoted to a preliminary description and investigation of the functions $Z(s, \sigma)$. We proceed analogously to [B, section (3.1)].

Let $\Phi$ be the geodesic flow on $S X$. Since $K$ acts transitively on the unit-sphere of $\mathfrak{p}$, there is a canonical isomorphism $S X \cong \Gamma \backslash G / M$ and in this way one obtains an isomorphism

$$
T S X \cong \Gamma \backslash G \times_{\text {Ad }}(\overline{\mathfrak{n}} \oplus \mathfrak{n} \oplus \mathfrak{a}) .
$$

We fix an $M$-invariant inner product on $\overline{\mathfrak{n}} \oplus \mathfrak{n} \oplus \mathfrak{a}$ and equip $T S X$ with the induced metric. With respect to (3.1), $\Phi$ is given on $\Gamma \backslash G / M$ as $\Phi(t, \Gamma g M)=\Gamma g \exp -t H_{1} M$, where $\Phi$ is well-defined since $M$ and $A$ commute. Thus, with respect to (3.1), $d \Phi$, regarded as a flow on $T S X$, is given by

$$
d \Phi(t,[\Gamma g, Y])=\left[\Gamma g \exp -t H_{1}, \operatorname{Ad}\left(\exp t H_{1}\right) Y\right], \quad Y \in \overline{\mathfrak{n}} \oplus \mathfrak{a} \oplus \mathfrak{n}, g \in G .
$$

The spaces $\overline{\mathfrak{n}}, \mathfrak{n}$ and $\mathfrak{a}$ are invariant under $\operatorname{Ad}(A)$ and $\operatorname{Ad}\left(\exp t H_{1}\right)$ acts on these spaces by $e^{-t} \cdot$ Id respectively $e^{t} \cdot \operatorname{Id}$ respectively Id. Thus the decomposition on the right hand side of (3.1) gives the Anosov-decomposition in (1.1).

Let $\sigma$ be a unitary finite dimensional representation of $M$ on $V_{\sigma}$ and let $V(\sigma):=\Gamma \backslash\left(G \times_{\sigma}\right.$ $\left.V_{\sigma}\right)$. Then $V(\sigma)$ is a vector bundle over $S X$ and $\Phi$ lifts to a flow $\Phi_{\sigma}$ on $V(\sigma)$ which is defined by $\Phi_{\sigma}(t,[\Gamma g, v]):=\left[\Gamma g \exp \left(-t H_{1}\right), v\right]$.

Next we describe the closed geodesics of $X$ in terms of the conjugacy classes of $\Gamma$ which we will denote by $\mathrm{C}(\Gamma)$. There is a canonical one-to one correspondence between $\mathrm{C}(\Gamma)$ and the set of free homotopy classes of closed paths in $X$. For $\gamma \in \Gamma$ we will denote its conjugacy class by $[\gamma]$. Moreover, by $f([\gamma])$ we will denote the free homotopy class of closed paths associated to $[\gamma]$. Now for $[\gamma] \in \mathrm{C}(\Gamma)$ we let $\ell(\gamma)$ be the infimum over all lengths of the piecewise smooth curves belonging to $f([\gamma])$. We let $\mathrm{C}(\Gamma)_{\mathrm{s}}$ be the set of conjugacy classes $[\gamma]$ such that $\gamma$ is semisimple. Moreover we let $\mathrm{C}(\Gamma)_{\text {par }}$ be the set of conjugacy classes $[\gamma]$ 
such that $\gamma$ is $\Gamma$-conjugate to an element of $\Gamma \cap N_{P}, P \in \mathfrak{P}$. Then by War, Lemma 5.3] and our assumption (1.3) we have $\mathrm{C}(\Gamma)=\mathrm{C}(\Gamma)_{\mathrm{s}} \cup \mathrm{C}(\Gamma)_{\text {par }}$ and $\mathrm{C}(\Gamma)_{\mathrm{s}} \cap \mathrm{C}(\Gamma)_{\text {par }}=[1]$. For $[\gamma] \in \mathrm{C}(\Gamma)_{\text {par }}$ it is easy to see that $\ell(\gamma)=0$.

On the other hand let $\gamma \in \Gamma$ be semimisple, $\gamma \neq 1$. Then by the same argument as in the proof of [Wal, Lemma 6.6] for the cocompact case, it follows that there exists $g \in G$, $t_{\gamma} \in(0, \infty)$ and $m_{\gamma} \in M$ such that $g \gamma g^{-1}=m_{\gamma} \exp t_{\gamma} H_{1}$, where $t_{\gamma}$ is unique and $m_{\gamma}$ is unique up to conjugation in $M$. Thus the geodesic $\widetilde{c}_{\gamma}(t):=g^{-1} \exp \left(t H_{1}\right) K$ in $\tilde{X}$ is stabilized by $\gamma$ and projects to a closed geodesic $c_{\gamma} \in f([\gamma])$ of length $t_{\gamma}$. Applying [BON, Proposition 4.2] we conclude that the elements in $\tilde{X}$ which minimize $d(x, \gamma x)$ are exactly the elements $\widetilde{c}_{\gamma}(t), t \in \mathbb{R}$. It follows that $\ell(\gamma)=t_{\gamma}$. Proceeding as in the proof of [Ga, Lemma 4.1], one can show that for every semisimple element $\gamma \in \Gamma$ its centralizer $Z(\gamma)$ in $\Gamma$ is infinite cyclic and generated by a semisimple element $\gamma_{0}$. Thus we may define $n_{\Gamma} \in \mathbb{N}$ by $\gamma=\gamma_{0}^{n_{\Gamma}(\gamma)}$. We call $\gamma$ resp. $[\gamma]$ prime if $n_{\Gamma}(\gamma)=1$. Then it is easy to see that this is equivalent to saying that $c_{\gamma}$ is a prime geodesic.

Putting everything together, it follows that the Selberg zeta function from equation (1.2) can be written as

$$
Z(s, \sigma)=\prod_{\substack{[\gamma] \in \mathrm{C}(\Gamma)_{s}-[1] \\[\gamma] \text { prime }}} \prod_{k=0}^{\infty} \operatorname{det}\left(\operatorname{Id}-\left.\sigma\left(m_{\gamma}\right) \otimes S^{k} \operatorname{Ad}\left(m_{\gamma} \exp \left(\ell(\gamma) H_{1}\right)\right)\right|_{\overline{\mathfrak{n}}} e^{-(s+n) \ell(\gamma)}\right) .
$$

In order to establish the convergence of the infinite product in (3.2), we remark that

$$
\operatorname{det}\left(\operatorname{Id}-\left.\operatorname{Ad}\left(m_{\gamma} a_{\gamma}\right)\right|_{\overline{\mathfrak{n}}}\right)^{-1} \leq\left(1-e^{-l(\gamma)}\right)^{-n} .
$$

Moreover, using the volume growth $\operatorname{vol} B_{R}(x) \leq c e^{2 n R}$ on $\tilde{X}$, the argument of MePo, Lemma 4.3, Proposition 4.4] carries over to higher dimensions and one obtains an estimate

$$
\#\left\{[\gamma] \in \mathrm{C}(\Gamma)_{\mathrm{s}}: \ell(\gamma) \leq R\right\} \leq C e^{2 n R}
$$

for all $R$ and some constant $C>0$. Now arguing as in BQ, equation (3.6)] one computes

$$
\log Z(s, \sigma)=-\sum_{[\gamma] \in \mathrm{C}(\Gamma)_{\mathrm{s}}-[1]} \frac{\operatorname{Tr}\left(\sigma\left(m_{\gamma}\right)\right) e^{-(s+n) \ell(\gamma)}}{n_{\Gamma}(\gamma) \operatorname{det}\left(\operatorname{Id}-\left.\operatorname{Ad}\left(m_{\gamma} a_{\gamma}\right)\right|_{\overline{\mathfrak{n}}}\right)}
$$

and applying (3.3) and (3.4) it follows that the infinte product in (1.2) resp. (3.2) converges absolutly and locally uniformly on the set $\operatorname{Re}(s)>2 n$.

The formula $[\mathrm{BO}$, equation (3.4)] for the logarithmic derivative of $R(s, \sigma)$ remains valid also in our case. A similar formula can also be obtained for the logarithmic derivative of $R(s, \tau)$. Thus it follows from (3.4) that the infinit products in (1.4) converges absolutely for $\operatorname{Re}(s)>2 n$ and that the infinite product in (1.5) converges absolutely for $s \in \mathbb{C}$ with $\operatorname{Re}(s)$ sufficiently large. Also, arguing as in cocompact case, [BO], Proposition 3.4], [Wo, section 6], we can express the functions $R(s, \sigma)$ and $R(s, \tau)$ as weighted products of Selberg zeta functions. 


\section{Some properties of the C-MAtrix}

In this section we describe some of the main properties of the $C$-matrix associated to the Eisenstein series which are needed for our application of the invariant trace formula. For $P \in \mathfrak{P}, \nu \in \hat{K}, \sigma_{P} \in \hat{M}_{P}$, with $\left[\nu: \sigma_{P}\right] \neq 0$ we let $\mathcal{E}_{P}\left(\nu, \sigma_{P}\right)$ be the set of all continuous functions $\Phi$ on $G$ which are left-invariant under $N_{P} A_{P}$ such that for all $x \in G$ the function $m \mapsto \Phi_{P}(m x)$ belongs to $L^{2}\left(M, \sigma_{P}\right)$, the $\sigma_{P}$-isotypical component of the right regular representation of $M_{P}$, and such that for all $x \in G$ the function $k \mapsto \Phi_{P}(x k)$ belongs to the $\nu$-isotypical component of the right regular representation of $K$. We define an inner product on $\mathcal{E}_{P}\left(\nu, \sigma_{P}\right)$ as follows. Every element of $\mathcal{E}_{P}\left(\nu, \sigma_{P}\right)$ can be identified canonically with a function on $K$. For $\Phi, \Psi \in \mathcal{E}_{P}\left(\nu, \sigma_{P}\right)$ we now set $\langle\Phi, \Psi\rangle:=\operatorname{vol}\left(\Gamma \cap N_{P} \backslash N_{P}\right) \int_{K} \Phi(k) \bar{\Psi}(k) d k$. Now we define a Hilbert space $\mathcal{E}_{P}\left(\sigma_{P}\right)$ by

$$
\mathcal{E}_{P}\left(\sigma_{P}\right):=\bigoplus_{\substack{\nu \in \hat{K} \\\left[\nu: \sigma_{P}\right] \neq 0}} \mathcal{E}_{P}\left(\nu, \sigma_{P}\right)
$$

For $\lambda \in \mathbb{C}$ let $\pi_{\Gamma, \sigma_{P}, \lambda}$ be the representation of $G$ on $\mathcal{E}_{P}\left(\sigma_{P}\right)$ defined by

$$
\pi_{\Gamma, \sigma_{P}, \lambda}(g) \Phi\left(n_{P} a_{P} k\right):=e^{(\lambda+n) H_{P}(k g)} \Phi(k g), \quad n_{P} \in N_{P}, a_{P} \in A_{P}, k \in K, \quad \Phi \in \mathcal{E}_{P}\left(\sigma_{P}, \nu\right) .
$$

For $\sigma \in \hat{M}$ and $\nu \in \hat{K}$ with $[\nu: \sigma] \neq 0$ put

$$
\mathcal{E}(\nu: \sigma):=\bigoplus_{\sigma_{P} \in \boldsymbol{\sigma}} \mathcal{E}\left(\nu: \sigma_{P}\right) ; \quad \mathcal{E}(\sigma):=\bigoplus_{\sigma_{P} \in \boldsymbol{\sigma}} \mathcal{E}_{P}\left(\sigma_{P}\right) ; \quad \pi_{\Gamma, \sigma, \lambda}:=\bigoplus_{\sigma_{P} \in \boldsymbol{\sigma}} \pi_{\Gamma, \sigma_{P}, \lambda}
$$

Then $\boldsymbol{\pi}_{\Gamma, \sigma, \lambda}$ is a representation of $G$ on $\mathcal{E}(\sigma)$. The pair $\left(\mathcal{E}(\sigma), \boldsymbol{\pi}_{\Gamma, \sigma, \lambda}\right)$ can be related to the the principal series as follows. For $\sigma \in \hat{M}$ let $\operatorname{Hom}_{M}\left(V_{\sigma}, L^{2}(M)\right)$ denote the set of intertwining operators between $\sigma$ and the right regular representation. The dimension of this space equals the degree of $\sigma$. For $\nu \in \hat{K}$ with $[\nu: \sigma] \neq 0$ let $\left(\mathcal{H}^{\sigma}\right)^{\nu}$ denote the $\nu$ isotypical component of $\mathcal{H}^{\sigma}$, where the latter space is as in section 2.5. Then we define $I_{P, \sigma}(\nu): \operatorname{Hom}_{M}\left(V_{\sigma}, L^{2}(M)\right) \otimes\left(\mathcal{H}^{\sigma}\right)^{\nu} \rightarrow \mathcal{E}_{P}\left(\sigma_{P}\right)$ by

$$
I_{P, \sigma}(u \otimes \Phi)(g):=u \circ \Phi\left(m k_{p}^{-1} \kappa_{P}(g)\right)\left(m^{-1}\right) \text { for almost all } m \in M .
$$

Then $I_{P, \sigma}(\nu)$ is an isometry. We let $I_{P, \sigma}$ be the direct sum of the $I_{P, \sigma}(\nu)$ and define

$$
\mathcal{L}(\sigma):=\bigoplus_{P \in \mathfrak{P}} \operatorname{Hom}_{M}\left(V_{\sigma}, L^{2}(M)\right) ; \quad \mathbf{I}_{\sigma}: \mathcal{L}(\sigma) \otimes \mathcal{H}^{\sigma} \longrightarrow \mathcal{E}(\sigma), \mathbf{I}_{\sigma}=\bigoplus_{P \in \mathfrak{P}} I_{P, \sigma}
$$

The map $\mathbf{I}_{\sigma}$ is an isomorphism and an intertwining operator between the representations $1 \otimes \pi_{\sigma, \lambda}$ and $\boldsymbol{\pi}_{\Gamma, \sigma, i \lambda}$, where 1 stands for the trivial representation of $G$ on $\mathcal{L}(\sigma)$.

Out of the constant terms associated to the Eisenstein series, one can construct operators

$$
\mathbf{C}(\nu: \sigma: \lambda): \mathcal{E}(\nu: \sigma) \rightarrow \mathcal{E}\left(\nu: w_{0} \sigma\right) ; \quad \mathbf{C}(\sigma: \lambda):=\bigoplus_{\substack{\nu \in \hat{K} \\[\nu: \sigma] \neq 0}} \mathbf{C}(\nu: \sigma: \lambda): \mathcal{E}(\sigma) \rightarrow \mathcal{E}\left(w_{0} \sigma\right)
$$


see [MP2, section 3]. For every $\sigma$ the function $\lambda \mapsto \mathbf{C}(\sigma: \lambda)$ is meromophic in $\lambda \in \mathbb{C}$ and has no poles on $i \mathbb{R}$. Moreover it satisfies the functional equation

$$
\mathbf{C}\left(w_{0} \sigma: \lambda\right) \mathbf{C}(\sigma:-\lambda)=\mathrm{Id} ; \quad \mathbf{C}(\sigma: \lambda)^{*}=\mathbf{C}\left(w_{0} \sigma: \bar{\lambda}\right) .
$$

The representations $\boldsymbol{\pi}_{\Gamma, \sigma, \lambda}$ and $\boldsymbol{\pi}_{\Gamma, w_{0} \sigma,-\lambda}$ are equivalent and $\mathbf{C}(\sigma: \lambda)$ is an intertwining operator between $\boldsymbol{\pi}_{\Gamma, \sigma, \lambda}$ and $\boldsymbol{\pi}_{\Gamma, w_{0} \sigma,-\lambda}$.

Let $\sigma \in \hat{M}$. According to [Ho2], the map $\mathbf{I}_{\sigma}$ can be used to relate the intertwining operators $\mathbf{C}(\sigma: s)$ to the Knapp-Stein intertwining operators of the principal series representations associated to $P_{0}$. One obtains a result similar to the adelic case, where the $\mathrm{C}$-matrix factorizes into a product of the Knapp-Stein intertwining operator and the intertwining operators at the finite places. We first briefly recall the definition of the Knapp-Stein operators. Let $\Theta$ denote the lift of $\theta$ to $G$, let $\bar{N}:=\Theta(N)$ and let $\bar{P}_{0}:=\bar{N} A K$ be the parabolic subgroup opposite to $P_{0}$. Let $\sigma \in \hat{M}$. For $\Phi \in \mathcal{H}^{\sigma}$ we define a function $\Phi_{\lambda}$ on $G$ by $\Phi_{\lambda}(n a k):=e^{(i \lambda+n) H(a)} \Phi(k)$. Let $\left(\mathcal{H}^{\sigma}\right)^{K}$ denote the $K$-finite vectors in $\mathcal{H}^{\sigma}$. Let $m_{0} \in K$ be a representative for $w_{0}$. Then for $\operatorname{Im}(\lambda)<0$ and $\Phi \in\left(\mathcal{H}^{\sigma}\right)^{K}$ the integral

$$
J_{\bar{P}_{0} \mid P_{0}}(\sigma, \lambda)(\Phi)(k):=\int_{\bar{N}} \Phi_{\lambda}(\bar{n} k) d \bar{n}=\int_{N} \Phi_{\lambda}\left(m_{0} n m_{0}^{-1} k\right) d n
$$

is convergent and $J_{\bar{P}_{0} \mid P_{0}}(\sigma, \lambda)$ extends to an intertwining operator $J_{\bar{P}_{0} \mid P_{0}}(\sigma, \lambda): \mathcal{H}^{\sigma} \longrightarrow \mathcal{H}^{\sigma}$ between $\pi_{\sigma, \lambda}$ and $\pi_{\sigma, \lambda, \bar{P}_{0}}$, where $\pi_{\sigma, \lambda, \bar{P}_{0}}$ denotes the principal series representation associated to $\sigma, \lambda$ and $\bar{P}_{0}$. Moreover, by $\left[\mathrm{KS}\right.$, as an operator-valued function $J_{\bar{P}_{0} \mid P_{0}}(\sigma, \lambda)$ has a meromorphic continuation to $\mathbb{C}$. If $\sigma \neq w_{0} \sigma, J_{\bar{P}_{0} \mid P_{0}}(\sigma, \lambda)$ has no poles on $i \mathbb{R}$ and is invertible there. If $\sigma=w_{0} \sigma, J_{\bar{P}_{0} \mid P_{0}}(\sigma, \lambda)$ is regular and invertible on $i \mathbb{R}-\{0\}$. Next one defines an operator $A\left(w_{0}\right): \mathcal{H}^{\sigma} \rightarrow \mathcal{H}^{w_{0} \sigma}$ by $A\left(w_{0}\right) \Phi(k):=\Phi\left(m_{0} k\right)$. Then $A\left(w_{0}\right)$ intertwines $\pi_{\sigma, \lambda, \bar{P}_{0}}$ and $\pi_{w_{0} \sigma,-\lambda}$. Thus the operator

$$
J_{P_{0}}(\sigma, \lambda): \mathcal{H}^{\sigma} \rightarrow \mathcal{H}^{w_{0} \sigma}, \quad J_{P_{0}}(\sigma, \lambda):=A\left(w_{0}\right) J_{\bar{P}_{0} \mid P_{0}}(\sigma, \lambda)
$$

is, wherever it is defined, an intertwining operator between $\pi_{\sigma, \lambda}$ and $\pi_{w_{0} \sigma,-\lambda}$. If $\nu \in \hat{K}$ with $[\nu: \sigma] \neq 0$ we denote by $J_{P_{0}}(\nu, \sigma, \lambda)$ the restriction of $J_{P_{0}}(\sigma, \lambda)$ to a map from $\left(\mathcal{H}^{\sigma}\right)^{\nu}$ to $\left(\mathcal{H}^{w_{0} \sigma}\right)^{\nu}$. Now the C-matrix is related to the Knapp-Stein operator as follows.

Proposition 4.1. There exist a meromorphic $\operatorname{Hom}\left(\mathcal{L}(\sigma), \mathcal{L}\left(w_{0} \sigma\right)\right)$-valued function $\mathbf{T}(\sigma, \lambda)$ which is regular on $i \mathbb{R}-\{0\}$ such that in the sense of meromorphic functions one has

$$
\mathbf{C}(\nu: \sigma: \lambda)\left(\mathbf{I}_{\sigma}(u \otimes \Phi)\right)=\mathbf{I}_{w_{0} \sigma}\left(\mathbf{T}(\sigma, \lambda)(u) \otimes J_{P_{0}}(\nu, \sigma,-i \lambda)(\Phi)\right)
$$

for all $u \in \mathcal{L}(\sigma)$ and all $\Phi \in\left(\mathcal{H}^{\sigma}\right)^{\nu}$.

Proof. The proposition is proved in [Ho2, Theorem 7.1] for the more general setting of a rank-one lattice and a Hecke operator.

Proposition 4.1 gives the following corollary. 
Corollary 4.2. Let $\alpha$ be a K-finite Schwarz-function. Then in the sense of meromorphic functions one has

$$
\begin{aligned}
& \operatorname{Tr}\left(\boldsymbol{\pi}_{\Gamma, \sigma, \lambda}(\alpha) \mathbf{C}(\sigma: \lambda)^{-1} \frac{d}{d \lambda} \mathbf{C}(\sigma: \lambda)\right)=\operatorname{Tr}\left(\mathbf{T}(\sigma, \lambda)^{-1} \frac{d}{d \lambda} \mathbf{T}(\sigma, \lambda)\right) \Theta_{\sigma,-i \lambda}(\alpha) \\
& -i \operatorname{dim}(\sigma) p \operatorname{Tr}\left(\pi_{\sigma,-i \lambda}(\alpha) J_{\bar{P}_{0} \mid P_{0}}(\sigma,-i \lambda)^{-1} \frac{d}{d z} J_{\bar{P}_{0} \mid P_{0}}(\sigma,-i \lambda)\right)
\end{aligned}
$$

Proof. We remark that since $\alpha$ is $K$-finite all traces are taken in finite-dimensional vector spaces. One has $\operatorname{dim}(\mathcal{L}(\sigma))=p \operatorname{dim}(\sigma)$ and by 4.4 ) one has

$$
J_{P_{0}}(\sigma,-i \lambda)^{-1} \frac{d}{d \lambda} J_{P_{0}}(\sigma,-i \lambda)=J_{\bar{P}_{0} \mid P_{0}}(\sigma,-i \lambda)^{-1} \frac{d}{d \lambda} J_{\bar{P}_{0} \mid P_{0}}(\sigma,-i \lambda) .
$$

Thus the corollary follows from Proposition 4.1 and the intertwining property of $\mathbf{I}_{\sigma}, \mathbf{I}_{w_{0} \sigma}$.

We shall now determine the logarithmic derivative of the function $\mathbf{T}(\sigma, \lambda)$. Let $\nu \in \hat{K}$ be a $K$-type of $\pi_{\sigma, \lambda}$. Then by section 2.5, $\nu$ occurs with mutliplicity 1 in $\pi_{\sigma, \lambda}$. Hence it follows from Schur's Lemma that

$$
\left.J_{\bar{P}_{0} \mid P_{0}}(\sigma, \lambda)\right|_{\left(\mathcal{H}^{\sigma}\right)^{\nu}}=c_{\nu}(\sigma: \lambda) \cdot \mathrm{Id},
$$

where $c_{\nu}(\sigma: \lambda) \in \mathbb{C}$. The function $\lambda \mapsto c_{\nu}(\sigma: \lambda)$ can be computed explicitly. If $k_{2}(\nu) e_{2}+$ $\cdots+k_{n+1}(\nu) e_{n+1}$ and $k_{2}(\sigma) e_{2}+\cdots+k_{n+1}(\sigma) e_{n+1}$ are the highest weight of $\nu$ resp. $\sigma$, by Theorem 8.2 in EKM one has, taking the different parametrization into account:

$$
c_{\nu}(\sigma: \lambda)=\alpha(n) \frac{\prod_{j=2}^{n+1} \Gamma\left(i \lambda-k_{j}(\sigma)-\rho_{j}\right) \prod_{j=2}^{n+1} \Gamma\left(i \lambda+k_{j}(\sigma)+\rho_{j}\right)}{\prod_{j=2}^{n+1} \Gamma\left(i \lambda-k_{j}(\nu)-\rho_{j}\right) \prod_{j=2}^{n+1} \Gamma\left(i \lambda+k_{j}(\nu)+\rho_{j}+1\right)},
$$

where $\alpha(n)$ is a constant depending only on $n$. Since $\operatorname{dim}(\mathcal{L}(\sigma))=p \operatorname{dim}(\sigma)$, it follows with Proposition 4.1, (4.5) and (4.6) that for every $\nu \in \hat{K}$ with $[\nu: \sigma] \neq 0$ one has

$$
\begin{aligned}
\operatorname{Tr}\left(\mathbf{T}(\sigma, i \lambda)^{-1} \frac{d}{d z} \mathbf{T}(\sigma, i \lambda)\right)= & \frac{1}{\operatorname{dim}(\nu)} \operatorname{Tr}\left(\mathbf{C}(\nu: \sigma: i \lambda)^{-1} \frac{d}{d z} \mathbf{C}(\nu: \sigma: i \lambda)\right) \\
& +i p \operatorname{dim}(\sigma) c(\nu: \sigma: \lambda)^{-1} \frac{d}{d \lambda} c(\nu: \sigma: \lambda) .
\end{aligned}
$$

Now for $\sigma \in \hat{M}$ with highest weight $k_{2}(\sigma) e_{2}+\cdots+k_{n+1}(\sigma) e_{n+1}$ we let $\nu_{\sigma} \in \hat{K}$ be the representation of $K$ with highest weight $k_{2}(\sigma) e_{2}+\cdots+\left|k_{n+1}(\sigma)\right| e_{n+1}$. Then by [GW], Theorem 8.1.4] we have $\left[\nu_{\sigma}: \sigma\right]=1$ and thus using (4.7) and (4.8) we get

$$
\begin{aligned}
\operatorname{Tr}\left(\mathbf{T}(\sigma, i \lambda)^{-1} \frac{d}{d z} \mathbf{T}(\sigma, i \lambda)\right)= & \frac{1}{\operatorname{dim}(\nu)} \operatorname{Tr}\left(\mathbf{C}\left(\nu_{\sigma}: \sigma: i \lambda\right)^{-1} \frac{d}{d z} \mathbf{C}\left(\nu_{\sigma}: \sigma: i \lambda\right)\right) \\
& +\sum_{j=2}^{n+1} \frac{p \operatorname{dim}(\sigma)}{i \lambda+\left|k_{j}(\sigma)\right|+\rho_{j}} .
\end{aligned}
$$

Finally we recall the factorization of the determinant of the $C$-matrix into an infinite product involving its zeroes and poles. Let $\sigma \in \hat{M}$ and $\nu \in \hat{K}$ with $[\nu: \sigma] \neq 0$. The 
restrictions of the representations $\pi_{\sigma, \lambda}$ and $\pi_{w_{0} \sigma,-\lambda}$ to $K$ are independent of the parameter $\lambda$ and are unitarily equivalent via the map $A\left(w_{0}\right): \mathcal{H}^{\sigma} \rightarrow \mathcal{H}^{w_{0} \sigma}$. If we tensor $A\left(w_{0}\right)^{-1}$ with an isometry $I^{\prime}(\sigma): \mathcal{L}\left(w_{0} \sigma\right) \rightarrow \mathcal{L}(\sigma)$ and use the isomorphisms $\mathbf{I}_{\sigma}$ and $\mathbf{I}_{w_{0} \sigma}$, we obtain an isometry $I(\sigma): \mathcal{E}\left(w_{0} \sigma\right) \rightarrow \mathcal{E}(\sigma)$ which maps $\mathcal{E}\left(w_{0} \sigma, \nu\right)$ to $\mathcal{E}(\sigma, \nu)$ for every $\nu \in \hat{K}$. Moreover by (4.4) and Proposition 4.1 for all $u \in \mathcal{L}(\sigma)$ and all $\Phi \in\left(\mathcal{H}^{\sigma}\right)^{\nu}$ we have

$$
I(\sigma) \circ \mathbf{C}(\nu: \sigma: \lambda) \circ \mathbf{I}_{\sigma}=\mathbf{I}_{\sigma} \circ\left(\left(I^{\prime}(\sigma) \circ \mathbf{T}(\sigma, \lambda)\right) \otimes J_{\bar{P}_{0} \mid P_{0}}(\nu, \sigma,-i \lambda)\right) .
$$

Thus using (4.6) it follows that the multiplicity of each pole of $\operatorname{det}(I(\sigma) \circ \mathbf{C}(\nu: \sigma: \lambda))$ is divisible by $\operatorname{dim}(\nu)$. Let $\{\beta\}$ and $\{\eta\}$ denote the set of poles of $\operatorname{det}(I(\sigma) \circ \mathbf{C}(\nu: \sigma: \lambda))$ on $(0, n]$ respectively $\{\lambda \in \mathbb{C}: \operatorname{Re}(\lambda)<0\}$, counted with multiplicity divided by $\operatorname{dim}(\nu)$. Then the set $\{\beta\}$ is finite and by $\mathbb{M u ̈ 2}$, Theorem 6.9] one has

$$
\begin{aligned}
& \frac{1}{\operatorname{dim}(\nu)} \operatorname{Tr}\left(\mathbf{C}(\nu: \sigma: \lambda)^{-1} \frac{d}{d s} \mathbf{C}(\nu: \sigma: \lambda)\right) \\
= & \log q(\sigma)+\sum_{\{\beta\}}\left(\frac{1}{\lambda+\beta}-\frac{1}{\lambda-\beta}\right)+\sum_{\eta}\left(\frac{1}{\lambda+\bar{\eta}}-\frac{1}{\lambda-\eta}\right),
\end{aligned}
$$

where $q(\sigma) \in \mathbb{R}^{+}$and where the sum converges absolutely.

Remark 4.3. Let $\nu \in \hat{K}$ and $\sigma \in \hat{M}$ with $[\nu: \sigma] \neq 0$. Then it follows from (4.2) that $\alpha$ is a pole of $\operatorname{det}(I(\sigma) \circ \mathbf{C}(\nu: \sigma: s))$ if and only if $\bar{\alpha}$ is a pole of $\operatorname{det}\left(I\left(w_{0} \sigma\right) \circ \mathbf{C}(\nu: \sigma: s)\right)$ and that the corresponding orders are equal.

\section{The InVARIANT TRACE FORMUla}

Let $\pi_{\Gamma}$ be the right-regular representation of $G$ on $L^{2}(\Gamma \backslash G)$. Then there exists an orthogonal decomposition

$$
L^{2}(\Gamma \backslash G)=L_{d}^{2}(\Gamma \backslash G) \oplus L_{c}^{2}(\Gamma \backslash G)
$$

of $L^{2}(\Gamma \backslash G)$ into closed $\pi_{\Gamma^{-}}$-invariant subspaces. The restriction of $\pi_{\Gamma}$ to $L_{d}^{2}(\Gamma \backslash G)$ decomposes into the orthogonal direct sum of irreducible unitary representations of $G$ and the multiplicity of each irreducible unitary representation of $G$ in this decomposition is finite. On the other hand, by the theory of Eisenstein series, the restriction of $\pi_{\Gamma}$ to $L_{c}^{2}(\Gamma \backslash G)$ is isomorphic to the direct integral over all unitary principle-series representations of $G$. These results are proved in [War, sections 1-3].

Now let $\alpha$ be a $K$-finite Schwarz function. Define an operator $\pi_{\Gamma}(\alpha)$ on $L^{2}(\Gamma \backslash G)$ by

$$
\pi_{\Gamma}(\alpha) f(x):=\int_{G} \alpha(g) f(x g) d g .
$$

Then relative to the decompostition (5.1) one has a splitting

$$
\pi_{\Gamma}(\alpha)=\pi_{\Gamma, d}(\alpha) \oplus \pi_{\Gamma, c}(\alpha) .
$$

It easily follows from $\left[\mathrm{Dg}\right.$, Theorem 9.1] that the operator $\pi_{\Gamma, d}(\alpha)$ is of trace class. In this section we recall the Selberg trace formula for $\operatorname{Tr}\left(\pi_{\Gamma, d}(\alpha)\right)$. First we introduce the distributions involved. Let $I(\alpha):=\operatorname{vol}(X) \alpha(1)$. By $[\mathrm{HC}$, Theorem 3], the Plancherel 
theorem can be applied to $\alpha$. Four groups of real rank one which do not possess a compact Cartan subgroup it is stated in $\mathbb{K n}$, Theorem 13.2]. Thus if $P_{\sigma}(z)$ is the Plancherel polynomial with respect to $\sigma$ as in $\mathbb{M P 2}$, equation (2.21)], one obtains

$$
I(\alpha)=\operatorname{vol}(X) \sum_{\sigma \in \hat{M}} \int_{\mathbb{R}} P_{\sigma}(i \lambda) \Theta_{\sigma, \lambda}(\alpha) d \lambda,
$$

where the sum is finite since $\alpha$ is $K$-finite. Next we define the semisimple contribution by

$$
H(\alpha):=\int_{\Gamma \backslash G} \sum_{\gamma \in \Gamma_{\mathrm{s}}-1} \alpha\left(x^{-1} \gamma x\right) d x .
$$

Here $\Gamma_{\mathrm{s}}$ are the semisimple elements of $\Gamma$. By War, Lemma 8.1] the integral converges absolutely. Its Fourier transform can be computed as follows. Let $\mathrm{C}(\Gamma)_{\mathrm{s}}$ be the set of semisimple conjugacy classes of $\Gamma$. For $[\gamma] \in \mathrm{C}(\Gamma)_{\mathrm{s}}-[1]$ let $m_{\gamma} \in M$ and $\ell(\gamma) \in \mathbb{R}^{+}$be as in section 3. Let $a_{\gamma}:=\exp \ell(\gamma) H_{1}$. Moreover let $\gamma_{0}$ be as in section 3. Then one puts

$$
L(\gamma, \sigma):=\frac{\overline{\operatorname{Tr}(\sigma)\left(m_{\gamma}\right)}}{\operatorname{det}\left(\operatorname{Id}-\left.\operatorname{Ad}\left(m_{\gamma} a_{\gamma}\right)\right|_{\mathfrak{n}}\right)} e^{-n \ell(\gamma)} .
$$

Then proceeding as in Wal, Chapter 6] and using [Ga, equation 4.6] one obtains

$$
H(\alpha)=\sum_{\sigma \in \hat{M}[\gamma] \in \mathrm{C}(\Gamma)_{\mathrm{s}}-[1]} \frac{l\left(\gamma_{0}\right)}{2 \pi} L(\gamma, \sigma) \int_{-\infty}^{\infty} \Theta_{\sigma, \lambda}(\alpha) e^{-i \ell(\gamma) \lambda} d \lambda
$$

where the sum is finite since $\alpha$ is $K$-finite.

Next let $P \in \mathfrak{P}$ and for every $\eta \in \Gamma \cap N_{P}-\{1\}$ let $X_{\eta}:=\log \eta$. Let $\|\cdot\|$ be the norm induced on $\mathfrak{n}_{P}$ by the restriction of $-\frac{1}{4 n} B(\cdot, \theta \cdot)$ to $\mathfrak{n}_{P}$. Then for $\operatorname{Re}(s)>0$ the Epstein zeta function $\zeta_{P}$ is defined by $\zeta_{P}(s):=\sum_{\eta \in \Gamma \cap N_{P}-\{1\}}\left\|X_{\eta}\right\|^{-2 n(1+s)}$. By [Ter, Chapter 1.4, Theorem 1], this series converges absolutely for $\operatorname{Re}(s)>0$ and $\zeta_{T}$ has a meromorphic continuation to $\mathbb{C}$ with a simple pole at $s=0$. Let $R_{P}(\Gamma), C_{P}(\Gamma)$ be the residue resp. the constant term of $\zeta_{P}$ at $s=0$. Then by [Ter, Chapter 1.4, Theorem 1] one has $R_{P}(\Gamma)=\frac{\operatorname{vol}\left(S^{2 n-1}\right)}{2 n \operatorname{vol}\left(\Gamma \cap N_{P} \backslash N_{P}\right)}$. Now for $C(\Gamma):=\sum_{P \in \mathfrak{P}} C_{P}(\Gamma) \operatorname{vol}\left(\Gamma \cap N_{P} \backslash N_{P}\right) / \operatorname{vol}\left(S^{2 n-1}\right)$ let

$$
T(\alpha):=C(\Gamma) \int_{K} \int_{N} \alpha\left(k n k^{-1}\right) d n ; \quad T_{P}^{\prime}(\alpha):=\int_{K} \int_{N_{P}} \alpha\left(k n_{P} k^{-1}\right) \log \left\|\log n_{P}\right\| d n_{P} d k .
$$

Then $T$ and $T_{P^{\prime}}$ are tempered distributions. The distributions $T$ is invariant. Applying the Fourier inversion formula and the Peter-Weyl-Theorem to equation 10.21 in [Kn], one obtains the Fourier transform of $\mathrm{T}$ as:

$$
T(\alpha)=\sum_{\sigma \in \hat{M}} \frac{\operatorname{dim}(\sigma)}{2 \pi} C(\Gamma) \int_{\mathbb{R}} \Theta_{\sigma, \lambda}(\alpha) d \lambda,
$$

see Wal, Lemma 6.3]. The distributions $T_{P}^{\prime}$ are not invariant. However, using the KnappStein intertwining operators, they can be made invariant as follows. Let $\epsilon>0$ be such that 0 is the only possible pole of the operators $J_{\bar{P}_{0} \mid P_{0}}(\sigma, z), J_{\bar{P}_{0} \mid P_{0}}(\sigma, z)^{-1}, \boldsymbol{T}(\sigma, z), \boldsymbol{T}(\sigma, z)^{-1}$ on $\{z \in \mathbb{C}:|z|<2 \epsilon\}$ for all $\sigma \in \hat{M}$ which satisfy $[\nu: \sigma] \neq 0, \nu$ a $K$-type of $\alpha$. Let $H_{\epsilon}$ be the 
half-circle from $-\epsilon$ to $\epsilon$ in the lower half-plane, oriented counter-clockwise. Let $D_{\epsilon}$ be the path which is the union of $(-\infty,-\epsilon], H_{\epsilon}$ and $[\epsilon, \infty)$. Let

$$
J_{\sigma}(\alpha):=-\frac{i p \operatorname{dim} \sigma}{4 \pi} \int_{D_{\epsilon}} \operatorname{Tr}\left(J_{\bar{P}_{0} \mid P_{0}}(\sigma, z)^{-1} \frac{d}{d z} J_{\bar{P}_{0} \mid P_{0}}(\sigma, z) \pi_{\sigma, z}(\alpha)\right) d z .
$$

The change of contour is only neccessary if $J_{\bar{P}_{0} \mid P_{0}}(\sigma, s)$ has a pole at 0 , i.e. if $\sigma=w_{0} \sigma$. Now we define a distribution $\mathcal{I}$ by

$$
\mathcal{I}(\alpha):=\sum_{P \in \mathfrak{P}} T_{P}^{\prime}(\alpha)+\sum_{\sigma \in \hat{M}} J_{\sigma}(\alpha) .
$$

Then by [Ho1], $\mathcal{I}$ is an invariant distribution, see [MP2]. Let

$$
\mathcal{S}(\alpha):=\frac{1}{4 \pi} \sum_{\sigma \in \hat{M}} \int_{D_{\epsilon}} \operatorname{Tr}\left(\boldsymbol{T}(\sigma, i z)^{-1} \frac{d}{d s} \boldsymbol{T}(\sigma, i z)\right) \Theta_{\sigma, z}(\alpha) d z,
$$

where $\boldsymbol{T}(\sigma, i z)$ is as in Proposition 4.1. Then the sum is finite since $\alpha$ is $K$-finite. By Corollary 4.2 we then have

$$
\sum_{\sigma \in \hat{M}} \frac{1}{4 \pi} \int_{\mathbb{R}} \operatorname{Tr}\left(\boldsymbol{\pi}_{\Gamma, \sigma, i \lambda}(\alpha) \mathbf{C}(\sigma: i \lambda)^{-1} \frac{d}{d z} \mathbf{C}(\sigma: i \lambda)\right) d \lambda=\mathcal{S}(\alpha)-J(\alpha) .
$$

Finally, the residual contribution is define by

$$
R(\alpha):=\sum_{\substack{\sigma \in \hat{M} \\ \sigma=w_{0} \sigma}}-\frac{1}{4} \operatorname{Tr}\left(\mathbf{C}(\sigma: 0) \boldsymbol{\pi}_{\Gamma, \sigma, 0}(\alpha)\right) .
$$

This sum is finite since $\alpha$ is $K$-finite. Using normalized intertwining operators, the Fourier transform of $R$ can be computed as follows. We use the notations of section 4 . For $\sigma \in \hat{M}, \sigma=w_{0} \sigma$ let $J_{\bar{P}_{0} \mid P_{0}}(\sigma, \lambda)$ be as in (4.3). Then $J_{\bar{P}_{0} \mid P_{0}}(\sigma, \lambda)$ might have a pole at $\lambda=0$. However, if the meromorphic function $r_{\bar{P}_{0} \mid P_{0}}(\sigma: \lambda)$ is defined as in Ho2, page 113-114], the map $R_{P_{0}}(\sigma: \lambda):=A\left(w_{0}\right) r_{\bar{P}_{0} \mid P_{0}}(\sigma: \lambda)^{-1} J_{\bar{P}_{0} \mid P_{0}}(\sigma: \lambda)$ is defined and invertible for $\lambda \in \mathbb{R}$, and it satisfies $R_{P_{0}}(\sigma: 0)^{-1}=R_{P_{0}}(\sigma: 0)$. By $\mathrm{KS}$, Proposition 49, Proposition 53] the representation $\pi_{\sigma, 0}$ is irreducible. Moreover, $R_{P_{0}}(\sigma: 0)$ satisfies $R_{P_{0}}(\sigma: 0) \circ \pi_{\sigma, 0}=\pi_{\sigma, 0} \circ R_{P_{0}}(\sigma: 0)$. Thus by [Kn, Corollary 8.13], $R_{P_{0}}(\sigma: 0)$ is a scalar operator. Thus one has $\left(R_{P_{0}}(\sigma: 0)\right)^{2}= \pm \mathrm{Id}$. Let $\mathbf{S}(\sigma: s):=r_{\bar{P}_{0} \mid P_{0}}(\sigma: s) \mathbf{T}(\sigma: s)$, where $\mathbf{T}(\sigma: s)$ is as in Proposition 4.1. Then $\mathbf{S}(\sigma: s)$ is a meromorphic $\operatorname{Hom}(\mathcal{L}(\sigma), \mathcal{L}(\sigma))$-valued function and since $\mathbf{C}(\sigma: 0)$ is defined and invertible, it follows from Proposition 4.1 that $\mathbf{S}(\sigma: s)$ is defined at $s=0$ and that $\mathbf{I}_{\sigma}^{-1} \mathbf{C}(\sigma: 0) \mathbf{I}_{\sigma}=\mathbf{S}(\sigma: 0) \otimes R_{P_{0}}(\sigma: 0)$, where $\mathbf{I}_{\sigma}$ is as in section 4 . Using the functional equation (4.2) one obtains $\mathbf{S}(\sigma: 0)^{*}=\mathbf{S}(\sigma: 0)$ and $\mathbf{S}(\sigma: 0)^{-1}=\mathbf{S}(\sigma: 0)$. Hence $\mathbf{S}(\sigma: 0)$ is diagonalizable with eigenvalues \pm 1 . Using the intertwining property of $\boldsymbol{I}_{\sigma}$, it follows that there exist natural numbers $c_{1}(\sigma), c_{2}(\sigma)$ with $c_{1}(\sigma)+c_{2}(\sigma)=p \operatorname{dim}(\sigma)$ such that one has

$$
-\frac{1}{4} \operatorname{Tr}\left(\mathbf{C}(\sigma: 0) \boldsymbol{\pi}_{\Gamma, \sigma, 0}(\alpha)\right)=\frac{c_{1}(\sigma)-c_{2}(\sigma)}{4} \Theta_{\sigma, 0}(\alpha)
$$


for every $K$-finite Schwarz function $\alpha$. We can now state the invariant trace formula.

Theorem 5.1. Let $\alpha$ be a K-finite Schwarz function. Then one has

$$
\operatorname{Tr}\left(\pi_{\Gamma, d}(\alpha)\right)=I(\alpha)+H(\alpha)+T(\alpha)+\mathcal{I}(\alpha)+R(\alpha)+\mathcal{S}(\alpha) .
$$

Proof. This theorem is a special case of the invariant trace formula stated in [Ho2, Theorem 6.4]. It follows if one combines [War, Theorem 8.4], the Theorem on page 299 in [OW], the explicit form of $R_{P}(\Gamma)$ and (5.7). Here one has to take into account that our normalizations are different from those of [OW].

The Fourier transform of the distribution $\mathcal{I}$ was computed in [Ho1]. We shall now state his result. Let $S\left(\mathfrak{b}_{\mathbb{C}}\right)$ be the symmetric algebra of $\mathfrak{b}_{\mathbb{C}}$. Define $\Pi \in S\left(\mathfrak{b}_{\mathbb{C}}\right)$ by $\Pi:=$ $\prod_{\alpha \in \Delta^{+}\left(\mathfrak{m}_{\mathbb{C}}, \mathfrak{b}_{\mathbb{C}}\right)} H_{\alpha}$. For $\sigma \in \hat{M}$ with highest weight $k_{2}(\sigma) e_{2}+\cdots+k_{n+1}(\sigma) e_{n+1}$ and $\lambda \in \mathbb{R}$ define $\lambda_{\sigma} \in(\mathfrak{h})_{\mathbb{C}}^{*}$ by $\lambda_{\sigma}:=i \lambda e_{1}+\sum_{j=2}^{n+1}\left(k_{j}(\sigma)+\rho_{j}\right) e_{j}$. We will denote by $\langle\cdot, \cdot\rangle$ the symmetric bilinear form on $\mathfrak{h}_{\mathbb{C}}^{*}$ induced by the Killing form. Then for $\alpha \in \Delta^{+}\left(\mathfrak{g}_{\mathbb{C}}, \mathfrak{h}_{\mathbb{C}}\right)$ we denote by $s_{\alpha}: \mathfrak{h}_{\mathbb{C}}^{*} \rightarrow \mathfrak{h}_{\mathbb{C}}^{*}$ the reflection $s_{\alpha}(x)=x-2 \frac{\langle x, \alpha\rangle}{\langle\alpha, \alpha\rangle} \alpha$. Now the Fourier transform of $\mathcal{I}$ is computed as follows.

Theorem 5.2. For every $K$-finite $\alpha \in \mathcal{C}^{2}(G)$ one has

$$
\mathcal{I}(\alpha)=\frac{p}{4 \pi} \sum_{\sigma \in \hat{M}} \int_{\mathbb{R}} \Omega(\check{\sigma},-\lambda) \Theta_{\sigma, \lambda}(\alpha) d \lambda,
$$

where the function $\Omega(\sigma, \lambda)$ is given by

$$
\Omega(\sigma, \lambda):=-2 \operatorname{dim}(\sigma) \gamma-\frac{1}{2} \sum_{\alpha \in \Delta^{+}\left(\mathfrak{g}_{\mathbb{C}}, \mathfrak{a}_{\mathbb{C}}\right)} \frac{\Pi\left(s_{\alpha} \lambda_{\sigma}\right)}{\Pi\left(\rho_{M}\right)}\left(\psi\left(1+\lambda_{\sigma}\left(H_{\alpha}\right)\right)+\psi\left(1-\lambda_{\sigma}\left(H_{\alpha}\right)\right) .\right.
$$

Here $\psi$ denotes the digamma function and $\gamma$ denotes the Euler-Mascheroni constant. Moreover $\check{\sigma}$ denotes the contragredient representation of $\sigma$.

Proof. This follows from [Ho1, Theorem 5], [Ho1, Theorem 6], Ho1, Corollary on page 96].

In order to show that the residues of the logarithmic derivative of the symmetrized Selberg zeta function are integral, we shall now take a closer look at the functions $\Omega(\sigma, \lambda)$. For $\sigma$ of highest weight $k_{2}(\sigma) e_{2}+\cdots+k_{n+1}(\sigma) e_{n+1}$ and $j=2, \ldots, n+1$ we let

$$
P_{j}(\sigma, \lambda)=\frac{\Pi\left(s_{e_{1}+e_{j}} \lambda_{\sigma}\right)}{\Pi\left(\rho_{M}\right)} .
$$

Then it was shown in MP2, equation 6.23] that

$$
P_{j}(\sigma, \lambda)=\operatorname{dim}(\sigma) \prod_{\substack{p=2 \\ p \neq j}}^{n+1} \frac{-\lambda^{2}-\left(k_{p}(\sigma)+\rho_{p}\right)^{2}}{\left(k_{j}(\sigma)+\rho_{j}\right)^{2}-\left(k_{p}(\sigma)-\rho_{p}\right)^{2}}
$$

Thus $P_{j}(\sigma, \lambda)$ is an even polynomial in $\lambda$ of degree $2 n-2$. Now the following lemma holds. 
Lemma 5.3. Let $j=2, \ldots, n+1$ and assume that all $k_{j}(\sigma)$ are integers with $k_{n+1}(\sigma) \geq 0$. Let $l \in \mathbb{N}$ with $k_{n+1}(\sigma) \leq l \leq k_{j}(\sigma)+\rho_{j}$. Then $P_{j}(\sigma, i l)$ is integral.

Proof. Suppose first that $l=k_{n+1}(\sigma)$. If $j<n+1$, one has $P_{j}(\sigma, i l)=0$ and if $j=n+1$, one has $P_{j}(\sigma, i l)=\operatorname{dim}(\sigma)$ by $(5.10)$.

Now suppose that $l>k_{n+1}(\sigma)$. Then there exists a minimal $\nu \in\{2, \ldots, n\}$ such that $l-\rho_{\nu} \geq k_{\nu+1}(\sigma)$. We have $\nu \geq j$. If $\nu>2$ we have $l-\rho_{\nu-1}<k_{\nu}(\sigma)$. Moreover, if $\nu>2$ with $l-\rho_{\nu-1}=k_{\nu}(\sigma)-1$ then $l=k_{\nu}(\sigma)+\rho_{\nu}$ and so in this case we have $P_{j}(\sigma, i l)=\operatorname{dim}(\sigma)$ for $\nu=j$ and $P_{j}(\sigma, i l)=0$ for $\nu>j$ by (5.10).

Thus it remains to consider the case that either $\nu=2$ or that for $\nu>2$ one has $k_{\nu}(\sigma)-1 \geq$ $l-\rho_{\nu-1}+1=l-\rho_{\nu}$. In this case we define $\Lambda^{\prime} \in \mathfrak{b}_{\mathbb{C}}^{*}$ by

$$
\Lambda^{\prime}:=\sum_{2 \leq i<j} k_{i}(\sigma) e_{i}+\sum_{j<i \leq \nu}\left(k_{i}(\sigma)-1\right) e_{i-1}+\left(l-\rho_{\nu}\right) e_{\nu}+\sum_{\nu<i \leq n+1} k_{i}(\sigma) e_{i} .
$$

Then $\Lambda^{\prime}$ is the highest weight $\Lambda\left(\sigma^{\prime}\right)$ of a representation $\sigma^{\prime}$ of $M$. By [MP2, equation 6.19], the restriction of $\left.s_{e_{1}+e_{j}} \lambda_{\sigma}\right|_{\lambda=i l}$ to $\mathfrak{b}_{\mathbb{C}}^{*}$ coincides with $\Lambda\left(\sigma^{\prime}+\rho_{M}\right)$ up to a permutation of the $e_{2}, \ldots, e_{n}$. Let $\xi \in \mathfrak{b}_{\mathbb{C}}^{*}, \xi=\xi_{2} e_{2}+\cdots+\xi_{n+1} e_{n+1}$. Then by [MP2, equation 6.18], if $\tau$ is any permutation of $\{2, \ldots, n+1\}$, for $\xi_{\tau}:=\xi_{2} e_{\tau(2)}+\cdots+\xi_{n+1} e_{\tau(n+1)}$, one has $\Pi\left(\xi_{\tau}\right)= \pm \Pi(\xi)$. Applying the Weyl dimension formula [GW, Theorem 7.19] one obtains

$$
\operatorname{dim}\left(\sigma^{\prime}\right)=\frac{\Pi\left(\Lambda\left(\sigma^{\prime}\right)+\rho_{M}\right)}{\Pi\left(\rho_{M}\right)}= \pm P_{j}(\sigma, i l) .
$$

This proves the lemma.

We can now state our main result about the function $\Omega(\sigma, \lambda)$.

Proposition 5.4. Let $\sigma \in \hat{M}$ with highest weight $k_{2}(\sigma) e_{2}+\cdots+k_{n+1}(\sigma) e_{n+1}$. Assume that all $k_{j}(\sigma)$ are integers. Let $m_{0}:=\left|k_{n+1}(\sigma)\right|$. Then there exists an even polynomial $Q(\sigma, \lambda)$ of degree $\leq 2 n-4$ and for every $j=2, \ldots, n+1$, every $l$ with $m_{0} \leq l<\left|k_{j}(\sigma)\right|+\rho_{j}$ there exist integers $c_{j, l}(\sigma)$ such that

$$
\begin{aligned}
\Omega(\sigma, \lambda)= & -\operatorname{dim}(\sigma)\left(2 \gamma+\psi(1+i \lambda)+\psi(1-i \lambda)+\sum_{1 \leq l<m_{0}} \frac{2 l}{\lambda^{2}+l^{2}}\right) \\
& -\sum_{j=2}^{n+1} \sum_{\substack{m_{0} \leq l<\\
\left|k_{j}(\sigma)\right|+\rho_{j}}} c_{j, l}(\sigma) \frac{2 l}{\lambda^{2}+l^{2}}-\sum_{j=2}^{n+1} \operatorname{dim}(\sigma) \frac{\left|k_{j}(\sigma)\right|+\rho_{j}}{\left(\left|k_{j}(\sigma)\right|+\rho_{j}\right)^{2}+\lambda^{2}}-Q(\sigma, \lambda) .
\end{aligned}
$$

For every $\sigma \in \hat{M}$ one has $\Omega(\sigma, \lambda)=\Omega\left(w_{0} \sigma, \lambda\right)=\Omega(\check{\sigma}, \lambda)$ and $Q(\sigma, \lambda)=Q\left(w_{0} \sigma, \lambda\right)$.

Proof. For $j=2, \ldots, n+1$ and $l \in \mathbb{N}$ with $m_{0} \leq l<\left|k_{j}(\sigma)\right|+\rho_{j}$ we let $c_{j, l}(\sigma):=P_{j}(\sigma, i l)$. Then $c_{j, l}(\sigma)$ is integral by Lemma 5.3. Thus if $\left|k_{n+1}(\sigma)\right|>0$, the proposition follows from [MP2, Proposition 6.4]. If $k_{n+1}(\sigma)=0$, the proof is exactly the same.

We remark that if all $k_{j}(\sigma)$ are half-integral, a statement analogous to Proposition 5.4 holds. For the proof one proceeds in the same way as above. 


\section{Bochner LAPLACE OPERATORS}

Let $\left(\nu, V_{\nu}\right) \in \hat{K}$ and let $\tilde{E}_{\nu}:=G \times_{\nu} V_{\nu}$ be the associated homogeneous vector bundle over $\widetilde{X}$ equipped with the metric induced from $V_{\nu}$. Then if one lets

$$
C^{\infty}(G, \nu):=\left\{f: G \rightarrow V_{\nu}: f \in C^{\infty}, f(g k)=\nu\left(k^{-1}\right) f(g), \forall g \in G, \forall k \in K\right\},
$$

the smooth sections of $\tilde{E}_{\nu}$ are canonically isomorphic to $C^{\infty}(G, \nu)$. A corresponding isometry also holds for the $L^{2}$-sections of $\tilde{E}_{\nu}$ and the space $L^{2}(G, \nu)$, which is defined analogously to (6.1). Let $\tilde{A}_{\nu}$ be the differential operator which is induced by $-\Omega$ on $C^{\infty}(G, \nu)$. Then by the same argument as in [MP2, section 4] $\tilde{A}_{\nu}$ with domain $C_{c}^{\infty}(G, \nu)$, the compactly supported elements of $C^{\infty}(G, \nu)$, is bounded from below and essentially selfadjoint. Its selfadjoint closure will be denoted by $\tilde{A}_{\nu}$ too. Let $e^{-t \tilde{A}_{\nu}}$ be the heat-semigroup of $\tilde{A}_{\nu}$ on $L^{2}(G, \nu)$. Then there exists a function

$$
H_{t}^{\nu}: G \longrightarrow \operatorname{End}\left(V_{\nu}\right) ; \quad H_{t}^{\nu}\left(k^{-1} g k^{\prime}\right)=\nu(k)^{-1} \circ H_{t}^{\nu}(g) \circ \nu\left(k^{\prime}\right), \forall k, k^{\prime} \in K, \forall g \in G
$$

such that

$$
\left(e^{-t \tilde{A}_{\nu}} \phi\right)(g)=\int_{G} H_{t}^{\nu}\left(g^{-1} g^{\prime}\right) \phi\left(g^{\prime}\right) d g^{\prime}, \quad \phi \in L^{2}(G, \nu), \quad g \in G,
$$

see [MP2, section 4]. By the arguments of [BM, Proposition 2.4], $H_{t}^{\nu}$ belongs to all HarishChandra Schwarz spaces $\left(\mathcal{C}^{q}(G) \otimes \operatorname{End}\left(V_{\nu}\right)\right), q>0$. We put $h_{t}^{\nu}(g):=\operatorname{tr} H_{t}^{\nu}(g)$, where $\operatorname{tr}$ denotes the trace in End $V_{\nu}$.

Now we pass to the quotient $X=\Gamma \backslash \widetilde{X}$. Let $E_{\nu}:=\Gamma \backslash \tilde{E}_{\nu}$ be the locally homogeneous bundle over $X$. Let $C_{c}^{\infty}(\Gamma \backslash G, \nu)$ resp. $L^{2}(\Gamma \backslash G, \nu)$ denote the $\Gamma$-left invariant elements of $C_{c}^{\infty}(G, \nu)$ resp. $L^{2}(G, \nu)$. Then $A_{\nu}$ with domain $C_{c}^{\infty}(\Gamma \backslash G, \nu)$ is essentially selfadjoint and bounded from below, see [MP2, section 4]. Its selfadjoint closure will be denoted by $A_{\nu}$ too. The decomposition (5.1) induces a decomposition of $L^{2}(\Gamma \backslash G, \nu) \cong\left(L^{2}(\Gamma \backslash G, \nu) \otimes V_{\nu}\right)^{K}$ as $L^{2}(\Gamma \backslash G, \nu)=L_{d}^{2}(\Gamma \backslash G, \nu) \oplus L_{c}^{2}(\Gamma \backslash G, \nu)$. This decomposition is invariant under $A_{\nu}$ in the sense of unbounded operators. Let $A_{\nu}^{d}$ denote the restriction of $A_{\nu}$ to $L_{d}^{2}(\Gamma \backslash G, \nu)$ in the sense of unbounded operators. Since $\pi_{\Gamma, d}$ decomposes discretely into a direct sum of irreducible unitary representations of $G$, there exists a subset $J \subset \mathbb{N}$, a sequence $\lambda_{j}, j \in J$ of real numbers and an orthonormal base $\phi_{j}, j \in J$ of $L_{d}^{2}(\Gamma \backslash G, \nu)$ such that $A_{\nu} \phi_{j}=\lambda_{j} \phi_{j}$ for every $j \in J$. The set $J$ may be finite. For $\lambda \in[0, \infty)$ let $N(\lambda):=\#\left\{j \in J: \lambda_{j} \leq \lambda\right\}$. By [D, Theorem 9.1], there exists a constant $C$ such that

$$
N(\lambda) \leq C(1+\lambda)^{\frac{d}{2}} .
$$

for every $\lambda$. Hence the operator $e^{-t A_{\nu}^{d}}$ is of trace class and one has

$$
\operatorname{Tr} \pi_{\Gamma, d}\left(h_{t}^{\nu}\right)=\operatorname{Tr}\left(e^{-t A_{\nu}^{d}}\right)=\sum_{j} e^{-t \lambda_{j}} .
$$

Using the methods of [BM], it was shown in [MP2, section 4] that

$$
\Theta_{\sigma, \lambda}\left(h_{t}^{\nu}\right)=e^{t\left(c(\sigma)-\lambda^{2}\right)}[\nu: \sigma] .
$$


for every $\sigma \in \hat{M}$. Here $c(\sigma)$ is as in section 2.5.

\section{The Symmetric Selberg Zeta function}

For $s \in \mathbb{C}, \operatorname{Re}(s)>2 n$ we define the symmetric Selberg zeta function $S(s, \sigma)$ by $S(s, \sigma):=Z(s, \sigma)$ if $\sigma=w_{0} \sigma$ and by by $S(s, \sigma):=Z(s, \sigma)+Z\left(s, w_{0} \sigma\right)$ if $\sigma \neq w_{0}$. In this section we want to study the function $S(s, \sigma)$.

By Proposition 2.1 there exist unique integers $m_{\nu}(\sigma) \in\{-1,0,1\}$ which are zero except for finitely many $\nu \in \hat{K}$ such that

$$
\sum_{\nu \in \hat{K}} m_{\nu}(\sigma) \iota^{*} \nu=\sigma \text {, if } \sigma=w_{0} \sigma ; \quad \sum_{\nu \in \hat{K}} m_{\nu}(\sigma) \iota^{*} \nu=\sigma+w_{0} \sigma, \text { if } \sigma \neq w_{0} \sigma .
$$

In the notations of section 6 , we define vector bundles $\tilde{E}(\sigma)$ and $E(\sigma)$ over $\tilde{X}$ resp. X by

$$
\tilde{E}(\sigma):=\bigoplus_{\substack{\nu \in \hat{K} \\ m_{\nu}(\sigma) \neq 0}} \tilde{E}_{\nu} ; \quad E(\sigma):=\bigoplus_{\substack{\nu \in \hat{K} \\ m_{\nu}(\sigma) \neq 0}} E_{\nu}
$$

Then $\tilde{E}(\sigma)$ and $E(\sigma)$ admit gradings $\tilde{E}(\sigma)=\tilde{E}^{+}(\sigma) \oplus \tilde{E}^{-}(\sigma)$ and $E(\sigma)=E^{+}(\sigma) \oplus E^{-}(\sigma)$ defined by the sign of $m_{\nu}(\sigma)$. For $\nu \in \hat{K}$ let $A_{\nu}$ and $A_{\nu}^{d}$ be as in section 6 and define

$$
A(\sigma):=\bigoplus_{\substack{\nu \in \hat{K} \\ m_{\nu}(\sigma) \neq 0}} A_{\nu}+c(\sigma) ; \quad A(\sigma)_{d}:=\bigoplus_{\substack{\nu \in \hat{K} \\ m_{\nu}(\sigma) \neq 0}} A_{\nu}^{d}+c(\sigma)
$$

where $c(\sigma)$ is as in section 2.5. Let $\tilde{A}(\sigma)$ be the lift of $A(\sigma)$ to $\tilde{E}(\sigma)$. Let

$$
h_{t}^{\sigma}(g):=e^{-t c(\sigma)} \sum_{\nu \in \hat{K}} m_{\nu}(\sigma) h_{t}^{\nu}(g),
$$

where $h_{t}^{\nu}$ is as in the previous section. Then by (7.1) and (6.6), for a principal series representation $\pi_{\sigma^{\prime}, \lambda}, \sigma^{\prime} \in \hat{M}, \lambda \in \mathbb{R}$ we have

$$
\Theta_{\sigma^{\prime}, \lambda}\left(h_{t}^{\sigma}\right)=e^{-t \lambda^{2}} \quad \text { for } \sigma^{\prime} \in\left\{\sigma, w_{0} \sigma\right\} ; \quad \Theta_{\sigma^{\prime}, \lambda}\left(h_{t}^{\sigma}\right)=0, \quad \text { otherwise. }
$$

Next we state a generalized resolvent formula which is due to Bunke and Olbrich.

Proposition 7.1. Let $s_{1}, \ldots, s_{N}$ be complex numbers with $s_{i}^{2} \neq s_{i^{\prime}}^{2}$ for $i \neq i^{\prime}$. Then for every $z \in \mathbb{C}-\left\{-s_{1}^{2}, \ldots,-s_{N}^{2}\right\}$ one has

$$
\sum_{i=1}^{N} \frac{1}{s_{i}^{2}+z} \prod_{\substack{i^{\prime}=1 \\ i^{\prime} \neq i}}^{N} \frac{1}{s_{i^{\prime}}^{2}-s_{i}^{2}}=\prod_{i=1}^{N} \frac{1}{s_{i}^{2}+z}
$$

Proof. This is proved by Bunke and Olbrich, [BO, Lemma 3.5].

Since the operators $A_{\nu}$ are bounded from below, there exists a $\lambda_{0}(\sigma) \in \mathbb{R}$ such that $A(\sigma) \geq \lambda_{0}(\sigma)$. Let $\lambda_{0}<\lambda_{1}<\ldots$ be the sequence of eigenvalues of $A(\sigma)$. This sequence might be either a finite or an infinte. For each $\lambda_{k}$ let $\mathcal{E}\left(\lambda_{k}\right)$ be the eigenspace of $A(\sigma)$ with eigenvalue $\lambda_{k}$ and define its graded dimension by $m_{\mathrm{s}}\left(\lambda_{k}, \sigma\right):=\operatorname{dim}_{\text {gr }} \mathcal{E}\left(\lambda_{k}\right)$. Now fix $N \in \mathbb{N}$ 
with $N>d / 2$ and choose distinct $s_{1}, \ldots, s_{N} \in \mathbb{C}$, such that $\operatorname{Re}\left(s_{i}\right)>2 n$ and such that $\operatorname{Re}\left(s_{i}^{2}\right)>\max \left\{0,-\lambda_{0}(\sigma)\right\}$ for all i. Then by Proposition 7.1 we have

$$
\sum_{k} m_{\mathrm{s}}\left(\lambda_{k}, \sigma\right) \prod_{i=1}^{N} \frac{1}{\lambda_{k}+s_{i}^{2}}=\int_{0}^{\infty} \sum_{i=1}^{N} \operatorname{Tr}_{\mathrm{s}} e^{-t\left(A(\sigma)_{d}+s_{i}^{2}\right)} \prod_{\substack{i^{\prime}=1 \\ i^{\prime} \neq i}}^{N} \frac{1}{s_{i^{\prime}}^{2}-s_{i}^{2}} d t
$$

where the super-trace is taken with respect to the grading defined above. The sum on the left hand side of (7.5) converges absolutely by (6.4). We compute the right hand side of (7.5) using the (6.5) and the invariant trace formula stated in Theorem 5.1. From now on we assume that in the weight $\Lambda(\sigma)$ of $\sigma$ as in (2.1) all $k_{i}(\sigma)$ are integers. If all $k_{i}(\sigma)$ are half-integral, one can proceed in the same way by making the appropriate modifications in the treatment of the distribution $\mathcal{I}$. To save notation we let $\epsilon(\sigma)=1$, if $\sigma=w_{0} \sigma$ and $\epsilon(\sigma)=2$, if $\sigma \neq w_{0} \sigma$.

We begin with the identity contribution. The polynomial $P_{\sigma}(\lambda)$ is even and of degree $d-1$ and by MP2, section 2.8] it satisfies $P_{\sigma}(\lambda)=P_{w_{0} \sigma}(\lambda)$. Thus by (5.2) and (7.4) one has

$$
\int_{0}^{\infty} \sum_{i=1}^{N} I\left(h_{t}^{\sigma}\right) e^{-t s_{i}^{2}} \prod_{\substack{i^{\prime}=1 \\ i^{\prime} \neq i}}^{N} \frac{1}{s_{i^{\prime}}^{2}-s_{i}^{2}} d t=\epsilon(\sigma) \pi \operatorname{vol}(X) \sum_{i=1}^{N} P_{\sigma}\left(s_{i}\right) \frac{1}{s_{i}} \prod_{\substack{i^{\prime}=1 \\ i^{\prime} \neq i}}^{N} \frac{1}{s_{i^{\prime}}^{2}-s_{i}^{2}}
$$

Next we come to the hyperbolic contribution. For $\gamma \in \mathrm{C}(\Gamma)_{\mathrm{s}}$ we let $L_{\mathrm{sym}}(\gamma, \sigma):=L(\gamma, \sigma)$, if $\sigma=w_{0} \sigma$ and $L_{\text {sym }}(\gamma, \sigma):=L(\gamma, \sigma)+L\left(\gamma, w_{0} \sigma\right)$, if $\sigma \neq w_{0} \sigma$. Here $L(\gamma, \sigma)$ is as in (5.3). Then using (5.4) and (7.4) we obtain

$$
\int_{0}^{\infty} \sum_{i=1}^{N} H\left(h_{t}^{\sigma}\right) e^{-t s_{i}^{2}} \prod_{\substack{i^{\prime}=1 \\ i^{\prime} \neq i}}^{N} \frac{1}{s_{i^{\prime}}^{2}-s_{i}^{2}} d t=\sum_{i=1}^{N} \sum_{[\gamma] \in \mathrm{C}(\Gamma)_{\mathrm{s}}-[1]} \ell\left(\gamma_{0}\right) L_{\mathrm{sym}}(\gamma, \sigma) e^{-s_{i} l(\gamma)} \frac{1}{2 s_{i}} \prod_{\substack{i^{\prime}=1 \\ i^{\prime} \neq i}}^{N} \frac{1}{s_{i^{\prime}}^{2}-s_{i}^{2}} .
$$

Since $\operatorname{Re}\left(s_{i}\right)>2 n$ for every i, the sum on the right hand side converges absolutely by equation (3.4) and equation (3.3). By GW, section 3.2.5] if $n$ is even we have $\check{\sigma}=\sigma$ and if $n$ is odd we have $\check{\sigma}=w_{0} \sigma$ for every $\sigma \in \hat{M}$. Thus, applying (3.5) we obtain

$$
\int_{0}^{\infty} \sum_{i=1}^{N} H\left(h_{t}^{\sigma}\right) e^{-t s_{i}^{2}} \prod_{\substack{i^{\prime}=1 \\ i^{\prime} \neq i}}^{N} \frac{1}{s_{i^{\prime}}^{2}-s_{i}^{2}} d t=\left.\sum_{i=1}^{N} \frac{d}{d s}\right|_{\substack{s=s_{i} \\ \text { a }}} \log S(s, \sigma) \frac{1}{2 s_{i}} \prod_{\substack{i^{\prime}=1 \\ i^{\prime} \neq i}}^{N} \frac{1}{s_{i^{\prime}}^{2}-s_{i}^{2}}
$$

For the distribution $T$ it follows from (5.5) and (7.4) that

$$
\int_{0}^{\infty} \sum_{i=1}^{N} T\left(h_{t}^{\sigma}\right) e^{-t s_{i}^{2}} \prod_{\substack{i^{\prime}=1 \\ i^{\prime} \neq i}}^{N} \frac{1}{s_{i^{\prime}}^{2}-s_{i}^{2}} d t=\frac{\epsilon(\sigma) \operatorname{dim}(\sigma)}{2} C(\Gamma) \sum_{i=1}^{N} \frac{1}{s_{i}} \prod_{\substack{i^{\prime}=1 \\ i^{\prime} \neq i}}^{N} \frac{1}{s_{i^{\prime}}^{2}-s_{i}^{2}}
$$


Using Theorem 5.2, Proposition 5.4, and equation (7.4) we compute:

$$
\begin{aligned}
& \int_{0}^{\infty} \sum_{i=1}^{N} \mathcal{I}\left(h_{t}^{\sigma}\right) e^{-t s_{i}^{2}} \prod_{\substack{i^{\prime}=1 \\
i^{\prime} \neq i}}^{N} \frac{1}{s_{i^{\prime}}^{2}-s_{i}^{2}} d t=-\frac{p \epsilon(\sigma)}{2} \sum_{j=2}^{n+1} \sum_{\substack{m_{0} \leq l \\
<\left|k_{j}(\sigma)\right|+\rho_{j}}} c_{j, l}(\sigma) \sum_{i=1}^{N} \frac{1}{l+s_{i}} \frac{1}{s_{i}} \prod_{\substack{i^{\prime}=1 \\
i^{\prime} \neq i}}^{N} \frac{1}{s_{i^{\prime}}^{2}-s_{i}^{2}} \\
& -\frac{p \epsilon(\sigma) \operatorname{dim}(\sigma)}{2} \sum_{i=1}^{N}\left(\gamma+\psi\left(1+s_{i}\right)+\sum_{1 \leq l<m_{0}} \frac{1}{l+s_{i}}\right) \frac{1}{s_{i}} \prod_{\substack{i^{\prime}=1 \\
i^{\prime} \neq i}}^{N} \frac{1}{s_{i^{\prime}}^{2}-s_{i}^{2}} \\
& -\frac{1}{4} \sum_{\substack{j=2 \\
\left|k_{j}(\sigma)\right|+\rho_{j}>0}}^{n+1} \sum_{i=1}^{N} \frac{p \epsilon(\sigma) \operatorname{dim}(\sigma)}{\left|k_{j}(\sigma)\right|+\rho_{j}+s_{i}} \frac{1}{s_{i}} \prod_{\substack{i^{\prime}=1 \\
i^{\prime} \neq i}}^{N} \frac{1}{s_{i^{\prime}}^{2}-s_{i}^{2}}-\frac{1}{4} \sum_{i=1}^{N} Q\left(\sigma, i s_{i}\right) \frac{p \epsilon(\sigma)}{s_{i}} \prod_{\substack{i^{\prime}=1 \\
i^{\prime} \neq i}}^{N} \frac{1}{s_{i^{\prime}}^{2}-s_{i}^{2}} .
\end{aligned}
$$

Next we treat the contribution of the distribution $\mathcal{S}$ defined in (5.6). For $\sigma \in \hat{M}$ let $\nu_{\sigma} \in \hat{K}$

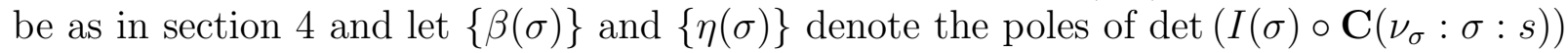
on $(0, n]$ and on $\{s \in \mathbb{C}: \operatorname{Re}(s)<0\}$, counted with multiplicity divided by $\operatorname{dim}\left(\nu_{\sigma}\right)$ as in section 1 . Then by (4.2), (4.9), (4.10) and (7.4) one has

$$
\begin{aligned}
& \int_{0}^{\infty} \sum_{i=1}^{N} \mathcal{S}\left(h_{t}^{\sigma}\right) e^{-t s_{i}^{2}} \prod_{\substack{i^{\prime}=1 \\
i^{\prime} \neq i}}^{N} \frac{1}{s_{i^{\prime}}^{2}-s_{i}^{2}} d t=\frac{\epsilon(\sigma)}{4} \sum_{\{\eta(\sigma)\}} \sum_{i=1}^{N}\left(\frac{1}{\eta(\sigma)-s_{i}}+\frac{1}{\bar{\eta}(\sigma)-s_{i}}\right) \frac{1}{s_{i}} \prod_{\substack{i^{\prime}=1 \\
i \neq i^{\prime}}}^{N} \frac{1}{s_{i^{\prime}}^{2}-s_{i}^{2}} \\
& +\frac{\epsilon(\sigma)}{2} \sum_{\{\beta(\sigma)\}} \sum_{i=1}^{N} \frac{1}{s_{i}+\beta(\sigma)} \frac{1}{s_{i}} \prod_{\substack{i^{\prime}=1 \\
i^{\prime} \neq i}}^{N} \frac{1}{s_{i^{\prime}}^{2}-s_{i}^{2}}+\frac{\epsilon(\sigma)}{4} \sum_{i=1}^{N} \log q(\sigma) \frac{1}{s_{i}} \prod_{\substack{i^{\prime}=1 \\
i^{\prime} \neq i}}^{N} \frac{1}{s_{i^{\prime}}^{2}-s_{i}^{2}} \\
& +\frac{\epsilon(\sigma) p \operatorname{dim}(\sigma)}{4} \sum_{j=2}^{n+1} \sum_{i=1}^{N} \frac{1}{\left|k_{j}(\sigma)\right|+\rho_{j}+s_{i}} \frac{1}{s_{i}} \prod_{\substack{i^{\prime}=1 \\
i^{\prime} \neq i}}^{N} \frac{1}{s_{i^{\prime}}^{2}-s_{i}^{2}} .
\end{aligned}
$$

Finally, the residual contribution is nonzero only if $\sigma=w_{0} \sigma$ and using (5.9) and (7.4) it follows that in this case we have

$$
\int_{0}^{\infty} \sum_{i=1}^{N} R\left(h_{t}^{\sigma}\right) e^{-t s_{i}^{2}} \prod_{\substack{i^{\prime}=1 \\ i^{\prime} \neq i}}^{N} \frac{1}{s_{i^{\prime}}^{2}-s_{i}^{2}} d t=\frac{c_{1}(\sigma)-c_{2}(\sigma)}{4} \sum_{i=1}^{N} \frac{1}{s_{i}^{2}} \cdot \prod_{i^{\prime}=1}^{N} \frac{1}{s_{i^{\prime}}^{2}-s_{i}^{2}}
$$

Now for $\sigma \neq w_{0} \sigma$ put $K(\sigma, s)=0$ and for $\sigma=w_{0} \sigma$ put $K(\sigma, s)=-\frac{c_{1}(\sigma)}{s}$. Moreover let $c_{\Gamma}(\sigma):=\epsilon(\sigma)(\operatorname{dim}(\sigma) C(\Gamma)-\operatorname{dim}(\sigma) \gamma p)$. Then the computations of this section can be summarized in the following proposition.

Proposition 7.2. For $s \in \mathbb{C}$ with $\operatorname{Re}(s)>2 n$ define

$$
\begin{aligned}
\Xi(s, \sigma):= & \exp \left(2 \pi \operatorname{vol}(X) \epsilon(\sigma) \int_{0}^{s} P_{\sigma}(r) d r-\epsilon(\sigma) \frac{p}{2} \int_{0}^{s} Q(\sigma, i r) d r+s c_{\Gamma}(\sigma)\right) \\
& \cdot(\Gamma(1+s))^{-p \epsilon(\sigma) \operatorname{dim}(\sigma)} \cdot S(s, \sigma) .
\end{aligned}
$$


Then there exist polynomials $C\left(\lambda_{k} ; s\right)$ and $C(\sigma ; s)$ in $s$ which are of degree at most $2(N-2)$ such that the logarithmic derivative of $\Xi$ satisfies

$$
\begin{aligned}
\frac{\Xi^{\prime}(s, \sigma)}{\Xi(s, \sigma)}= & 2 s \sum_{k} m_{\mathrm{s}}\left(\lambda_{k}, \sigma\right)\left(\frac{1}{s^{2}+\lambda_{k}}+C\left(\lambda_{k} ; s\right)\right)+\sum_{1 \leq l<m_{0}} p \epsilon(\sigma) \operatorname{dim}(\sigma) \frac{1}{l+s} \\
& +\sum_{j=2}^{n+1} \sum_{\substack{m_{0} \leq l<\\
\left|k_{j}(\sigma)\right|+\rho_{j}}} p \epsilon(\sigma) c_{j, l}(\sigma) \cdot \frac{1}{l+s}+\sum_{\eta(\sigma)} \frac{\epsilon(\sigma)}{2}\left(\frac{1}{s-\eta(\sigma)}+\frac{1}{s-\bar{\eta}(\sigma)}\right) \\
& -\sum_{\beta(\sigma)} \frac{\epsilon(\sigma)}{s+\beta(\sigma)}-\frac{\epsilon(\sigma) \log q(\sigma)}{2}+K(\sigma, s)+s C(\sigma ; s),
\end{aligned}
$$

where all involved sums converge absolutely.

Proof. We let $s_{1}=: s$ and fix $s_{2}, \ldots, s_{N}$. If we multiply (7.5) by $2 s \prod_{i^{\prime}=2}^{N}\left(s_{i^{\prime}}^{2}-s^{2}\right)$ and use that for $\sigma=w_{0} \sigma$ we have $k_{n+1}(\sigma)=0$ and $c_{1}(\sigma)+c_{2}(\sigma)=p \operatorname{dim}(\sigma)$, the Lemma follows from Propositon 7.1 and from the above computations.

\section{Twisted Dirac Operators}

In this section we introduce certain twisted Dirac operators on $\widetilde{X}$ and compute the Fourier transform of the corresponding heat-kernel. We keep the notations of section 2.4. Let $\mathrm{Cl}(\mathfrak{p})$ be the Clifford-algebra of $\mathfrak{p}$ with respect to the scalar product on $\mathfrak{p}$ defined in section 2.2. Let $\mathrm{Cl}(\widetilde{X}):=G \times{ }_{\mathrm{Ad}} \mathrm{Cl}(\mathfrak{p})$ be the Clifford bundle over $\widetilde{X}$. Let $\tilde{S}=G \times{ }_{\kappa} \Delta^{2 n}$ be the spinor bundle of $\widetilde{X}$. We denote by c : $\mathrm{Cl}(\mathfrak{p}) \otimes \Delta^{2 n} \longrightarrow \Delta^{2 n}, X \otimes v \mapsto \mathrm{c}(X) v$ the Clifford multiplication. Since $M$ centralizes $\mathfrak{a}$, there is an $\epsilon \in\{ \pm 1\}$ such that $\epsilon \mathrm{c}\left(H_{1}\right)$ acts on the spaces $\Delta_{ \pm}^{2 n}$ with eigenvalues $\mp i$, where $H_{1}$ is in section 2.2.

Now let $\sigma \in \hat{M}$ and assume that $\sigma \neq w_{0} \sigma, k_{n+1}(\sigma)>0$. Let $\nu(\sigma) \in \hat{K}$ be as in Proposition 2.1. Let $\tilde{E}(\sigma)$ be the vector bundle over $\tilde{X}$ as in section 7 . Then by Proposition 2.1 one has $\tilde{E}(\sigma)=\tilde{E}_{\nu(\sigma)} \otimes \tilde{S}$. We denote by $\tilde{D}(\sigma)$ the twisted Dirac-Operator on $\tilde{E}(\sigma)$, multiplied by $\epsilon$. Then we have the following proposition.

Proposition 8.1. Let $\tilde{A}(\sigma)$ be as in section 7 . Then one has $\tilde{A}(\sigma)=\tilde{D}(\sigma)^{2}$.

Proof. We regard $\tilde{D}(\sigma)$ as an operator on $C^{\infty}(G, \nu(\sigma) \otimes \kappa)$, where the latter space is as in (6.1). In [AS], it was assumed that $\operatorname{rk}(G)=\operatorname{rk}(K)$. However, one can proceed exactly as in [AS, page 53-54] to obtain $\tilde{D}(\sigma)^{2}=-\Omega+\left(\nu(\sigma)\left(\Omega_{K}\right)-\kappa\left(\Omega_{K}\right)\right)$ Id, which is analogous to AS, equation (A 9)]. The highest weight $\Lambda(\nu(\sigma))$ of $\nu(\sigma)$ is as in Proposition 2.1 and the highest weight of $\kappa$ is given by $\Lambda(\kappa)=\frac{1}{2} e_{2}+\ldots+\frac{1}{2} e_{n+1}$. Thus applying [MP1, equation 2.18] one computes $\nu(\sigma)\left(\Omega_{K}\right)-\kappa\left(\Omega_{K}\right)=c(\sigma)$ and the Lemma is proved.

Then $\tilde{D}(\sigma)$ and $\tilde{D}(\sigma)^{2}$ with domain $C_{c}^{\infty}(G, \nu(\sigma) \otimes \kappa) \subset L^{2}(G, \nu(\sigma) \otimes \kappa)$ are essentially selfadjoint, see [LM], Theorem II.5.7] and [MP2, section 4]. Their selfajoint closures will be denoted by the same symbols. Let $X_{1}, \ldots, X_{2 n+1}$ be an orthonormal base of $\mathfrak{p}$. Then by 
(6.3) $\tilde{D}(\sigma) e^{-t \tilde{D}(\sigma)^{2}}$ acts on $L^{2}(G, \nu(\sigma) \otimes \kappa)$ as a convolution operator with smooth kernel

$$
K_{t}^{\sigma}(g):=\left.\epsilon e^{-t c(\sigma)} \sum_{i=1}^{2 n+1} \operatorname{Id} \otimes \mathrm{c}\left(X_{i}\right) \circ \frac{d}{d t}\right|_{t=0} H_{t}^{\nu(\sigma) \otimes \kappa}\left(\exp -t X_{i} g\right),
$$

where $H_{t}^{\nu(\sigma) \otimes \kappa}$ is as in 6.2). Since $H_{t}^{\nu(\sigma) \otimes \kappa}$ belongs to all Harish-Chandra Schwarz spaces $\left(\mathcal{C}^{q}(G) \otimes \text { End }\left(V_{\nu(\sigma)} \otimes \Delta^{2 n}\right)\right)^{K}, q>0$ and since these spaces are stable under the differential action of $U\left(\mathfrak{g}_{\mathbb{C}}\right)$, it follows that $K_{t}^{\nu(\sigma)} \in\left(\mathcal{C}^{q}(G) \otimes \operatorname{End}\left(V_{\nu(\sigma)} \otimes \Delta^{2 n}\right)\right)^{K}$. Define a $K$-finite Schwarz function $k_{t}^{\sigma}:=\operatorname{Tr} K_{t}^{\sigma}$, where $\operatorname{Tr}$ is the trace in End $\left(V_{\nu(\sigma)} \otimes \Delta^{2 n}\right)$. Then the Fourier transform of $k_{t}^{\sigma}$ was computed by Moscovici and Stanton [MS]. It is given as follows.

Proposition 8.2. Let $\sigma \in \hat{M}, k_{n+1}(\sigma)>0$. Then for $\lambda \in \mathbb{R}$ one has

$$
\Theta_{\sigma, \lambda}\left(k_{t}^{\sigma}\right)=(-1)^{n} \lambda e^{-t \lambda^{2}} ; \quad \Theta_{w_{0} \sigma, \lambda}\left(k_{t}^{\sigma}\right)=(-1)^{n+1} \lambda e^{-t \lambda^{2}} .
$$

Moreover, if $\sigma^{\prime} \in \hat{M}, \sigma^{\prime} \notin\left\{\sigma, w_{0} \sigma\right\}$, for every $\lambda \in \mathbb{R}$ one has $\Theta_{\sigma^{\prime}, \lambda}\left(k_{t}^{\sigma}\right)=0$.

Proof. Let $\pi$ be an admissible unitary representation of $G$ on $\mathcal{H}_{\pi}$. Define an operator $\tilde{D}_{\sigma}(\pi)$ on the $K$-invariant subspace $\left(\mathcal{H}_{\pi} \otimes V_{\nu(\sigma)} \otimes \Delta^{2 n}\right)^{K}$ by $\tilde{D}_{\sigma}(\pi):=\epsilon \sum_{i=1}^{2 n+1} \pi\left(X_{i}\right) \otimes \operatorname{Id} \otimes \mathrm{c}\left(X_{i}\right)$. Then combining the arguments of [BM], [MP2, section 4] and Proposition 8.1 one easily obtains $\operatorname{Tr}\left(\pi\left(k_{t}^{\sigma}\right)\right)=e^{t(\pi(\Omega)-c(\sigma))} \operatorname{Tr} \tilde{D}_{\sigma}(\pi)$. Now by [MS, Proposition 3.6], where an $i$ has to be added by the arguments of Chapter 3 in [MS, it follows that for every $\sigma^{\prime} \in \hat{M}$ one has $\operatorname{Tr} \tilde{D}_{\sigma}\left(\pi_{\sigma^{\prime}, \lambda}\right)=\lambda\left(\operatorname{dim}\left(V_{\sigma^{\prime}} \otimes V_{\nu(\sigma)} \otimes \Delta_{+}^{2 n}\right)^{M}-\operatorname{dim}\left(V_{\sigma}^{\prime} \otimes V_{\nu(\sigma)} \otimes \Delta_{-}^{2 n}\right)^{M}\right)$. Let $\check{\sigma}$ be the contragredient representation of $\sigma$. By [GW], section 3.2.5] if $n$ is even one has $\check{\sigma}=\sigma$ and if $n$ is odd one has $\check{\sigma}=w_{0} \sigma$. Hence by Proposition 2.1, for $\sigma^{\prime} \in \hat{M}$ and $\lambda \in \mathbb{R}$ one has $\operatorname{dim}\left(V_{\sigma^{\prime}} \otimes V_{\nu(\sigma)} \otimes \Delta_{+}^{2 n}\right)^{M}-\operatorname{dim}\left(V_{\sigma^{\prime}} \otimes V_{\nu(\sigma)} \otimes \Delta_{-}^{2 n}\right)^{M}=(-1)^{n}\left[\sigma-w_{0} \sigma: \sigma^{\prime}\right]$. If we apply the formula for $\pi_{\sigma, \lambda}(\Omega)$ from section 2.5, the Proposition follows.

\section{The antisymmetric Selberg Zeta function}

Let $\sigma \in \hat{M}$ with $k_{n+1}(\sigma)>0$. Let $E(\sigma)=\Gamma \backslash \tilde{E}(\sigma)$ be the locally homogeneous vector bundle over $X$ as in section 7 . Let $\tilde{D}(\sigma)$ be as in the previous section. Then $\tilde{D}(\sigma)$ pushes down to an operator $D(\sigma)$ on the smooth sections of $E(\sigma)$. By Proposition 8.1 one has $D(\sigma)^{2}=A(\sigma)$, where $A(\sigma)$ is the operator from section 7 . The operator $D(\sigma)$ has a well defined restriction to $L_{d}^{2}(\Gamma \backslash G, \nu(\sigma) \otimes \kappa)$ in the sense of unbounded operators. This restriction will be denoted by $D(\sigma)_{d}$. The space $L_{d}^{2}(\Gamma \backslash G, \nu(\sigma) \otimes \kappa)$ is an orthogonal direct sum of finite dimensional eigenspaces of $D(\sigma)_{d}^{2}$. On these spaces $D(\sigma)_{d}$ acts as a symmetric operator, and thus $L_{d}^{2}(\Gamma \backslash G, \nu(\sigma) \otimes \kappa)$ is also the orthogonal direct sum of eigenspaces of $D(\sigma)_{d}$. By (6.4) the operator $D(\sigma)_{d} e^{-t D(\sigma)_{d}^{2}}$ is of trace class. One has

$$
\operatorname{Tr}\left(D(\sigma)_{d} e^{-t D(\sigma)_{d}^{2}}\right)=\operatorname{Tr}\left(\pi_{\Gamma, d}\left(k_{t}^{\sigma}\right)\right),
$$

where $k_{t}^{\sigma}$ is as in the previous section. Let $\left\{\mu_{k}\right\}$ be a fixed sequence of the eigenvalues of $D(\sigma)_{d}$ with $\mu_{k}=\mu_{k^{\prime}}$ iff $k=k^{\prime}$. This sequence might be finite or infinite. Let $\mathcal{E}\left(\mu_{k}\right)$ be the 
eigenspace of $D(\sigma)$ corresponding to the eigenvalue $\mu_{k}$ and let $d\left(\mu_{k}, \sigma\right):=\operatorname{dim}\left(\mathcal{E}\left(\mu_{k}\right)\right)$. Let $N \in \mathbb{N}, N>n+1$. We choose distinct points $s_{1}, \ldots, s_{N}$ such that $\operatorname{Re}\left(s_{j}\right)>2 n, \operatorname{Re}\left(s_{j}^{2}\right)>0$ for all j. By Proposition 7.1 we have

$$
2 i \sum_{k} d\left(\mu_{k}, \sigma\right) \mu_{k} \prod_{j=1}^{N} \frac{1}{\mu_{k}^{2}+s_{j}^{2}}=2 i \int_{0}^{\infty} \sum_{j=1}^{N} e^{-t s_{j}^{2}} \operatorname{Tr}\left(D(\sigma)_{d} e^{-t D(\sigma)_{d}^{2}}\right) \prod_{\substack{j^{\prime}=1 \\ j^{\prime} \neq j}}^{N} \frac{1}{s_{j^{\prime}}^{2}-s_{j}^{2}} d t .
$$

The sum on the left hand side converges absoluetly by (6.4).

We compute the right hand side of (9.2) using (9.1) and the invariant trace formula stated in Theorem 5.1. By [MP2, equation (2.22)] we have $P_{\sigma}(\lambda)=P_{w_{0} \sigma}(\lambda)$. By Proposition 5.4 we have $\Omega(\sigma, \lambda)=\Omega\left(w_{0} \sigma, \lambda\right)$. Thus, applying (5.2), (5.5) (5.9), Theorem 5.2 and Proposition 8.2, it follows that $I\left(k_{t}^{\sigma}\right), T\left(k_{t}^{\sigma}\right), \mathcal{I}\left(k_{t}^{\sigma}\right)$ and $R\left(k_{t}^{\sigma}\right)$ vanish.

If $L(\gamma, \sigma), L\left(\gamma, w_{0} \sigma\right)$ are as in (5.3), it follows from (5.4) and Proposition 8.2 that

$$
\begin{aligned}
& 2 i \int_{0}^{\infty} \sum_{j=1}^{N} e^{-t s_{j}^{2}} H\left(k_{t}^{\sigma}\right) \prod_{\substack{j^{\prime}=1 \\
j^{\prime} \neq j}}^{N} \frac{1}{s_{j^{\prime}}^{2}-s_{j}^{2}} d t \\
& =(-1)^{n} \sum_{j=1}^{N} \sum_{[\gamma] \in \mathrm{C}(\Gamma)_{\mathrm{s}}-[1]} l\left(\gamma_{0}\right)\left(L(\gamma, \sigma)-L\left(\gamma, w_{0} \sigma\right)\right) e^{-l(\gamma) s_{j}} \prod_{\substack{j^{\prime}=1 \\
j^{\prime} \neq j}}^{N} \frac{1}{s_{j^{\prime}}^{2}-s_{j}^{2}} .
\end{aligned}
$$

For $\operatorname{Re}(s)>2 n$ we define the anti-symmetric Selberg-zeta function by $S_{a}(s, \sigma):=\frac{Z(s, \sigma)}{Z\left(s, w_{0} \sigma\right)}$. As above, by GW, section 3.2.5] if $n$ is even we have $\overline{\operatorname{Tr}(\sigma)}=\operatorname{Tr}(\sigma)$ and if $n$ is odd we have $\overline{\operatorname{Tr}(\sigma)}=\operatorname{Tr}\left(w_{0} \sigma\right)$ for every $\sigma \in \hat{M}$. Thus using (3.5) we obtain

$$
2 i \int_{0}^{\infty} \sum_{j=1}^{N} e^{-t s_{j}^{2}} H\left(k_{t}^{\sigma}\right) \prod_{\substack{j^{\prime}=1 \\ j^{\prime} \neq j}}^{N} \frac{1}{s_{j^{\prime}}^{2}-s_{j}^{2}} d t=\left.\sum_{j=1}^{N} \frac{d}{d s}\right|_{s=s_{j}} \log S_{a}(s, \sigma) \prod_{\substack{j^{\prime}=1 \\ j^{\prime} \neq j}}^{N} \frac{1}{s_{j^{\prime}}^{2}-s_{j}^{2}} .
$$

Now we treat the distribution $\mathcal{S}$. Let $\eta(\sigma), \eta\left(w_{0} \sigma\right)$ be the poles of $\operatorname{det}\left(I(\sigma) \circ \mathbf{C}\left(\nu_{\sigma}: \sigma: s\right)\right)$ resp. of $\operatorname{det}\left(I\left(w_{0} \sigma\right) \circ \mathbf{C}\left(\nu_{\sigma}: w_{0} \sigma: s\right)\right)$ with negative real part and with multiplicity by $\operatorname{dim}\left(\nu_{\sigma}\right)$ as in section 1 . Then by (4.9), (4.10), Remark 4.3 and Proposition 8.2 one has

$$
\begin{aligned}
2 i \int_{0}^{\infty} \sum_{j=1}^{N} e^{-t s_{j}^{2} S\left(k_{t}^{\sigma}\right)} \prod_{\substack{j^{\prime}=1 \\
j^{\prime} \neq j}}^{N} \frac{1}{s_{j^{\prime}}^{2}-s_{j}^{2}} d t=(-1)^{n+1} \sum_{\eta(\sigma)} \sum_{j=1}^{N} \frac{1}{s_{j}-\eta(\sigma)} \prod_{\substack{j^{\prime}=1 \\
j^{\prime} \neq j}}^{N} \frac{1}{s_{j^{\prime}}^{2}-s_{j}^{2}} \\
+(-1)^{n} \sum_{\eta\left(w_{0} \sigma\right)} \sum_{j=1}^{N} \frac{1}{s_{j}-\eta\left(w_{0} \sigma\right)} \prod_{\substack{j^{\prime}=1 \\
j^{\prime} \neq j}}^{N} \frac{1}{s_{j^{\prime}}^{2}-s_{j}^{2}} .
\end{aligned}
$$

Let $\sigma \in \hat{M}$ with $k_{n+1}(\sigma)<0$. Then we define the twisted Dirac operator $D(\sigma)$ as $D(\sigma):=$ $-D\left(w_{0} \sigma\right)$, where $D\left(w_{0} \sigma\right)$ is as in section 8. We can now prove our main result about the antisymmetric Selberg zeta function. 
Proposition 9.1. The antisymmetric Selberg zeta function $S_{a}(s, \sigma)$ satisfies

$$
\begin{aligned}
\frac{S_{a}^{\prime}(s, \sigma)}{S_{a}(s, \sigma)}= & \sum_{k}\left(\frac{d\left(\mu_{k}, \sigma\right)}{s-i \mu_{k}}-\frac{d\left(\mu_{k}, \sigma\right)}{s+i \mu_{k}}+C\left(\mu_{k} ; s\right)\right)+(-1)^{n} \sum_{\eta(\sigma)}\left(\frac{1}{s-\eta(\sigma)}+C^{\prime}(\eta(\sigma) ; s)\right. \\
& +(-1)^{n+1} \sum_{\eta\left(w_{0} \sigma\right)}\left(\frac{1}{s-\eta\left(w_{0} \sigma\right)}+C^{\prime}\left(\eta\left(w_{0} \sigma\right) ; s\right)\right),
\end{aligned}
$$

where $C\left(\mu_{k} ; s\right), C(\eta(\sigma) ; s)$ and $C\left(\eta\left(w_{0} \sigma\right) ; s\right)$ are polynomials in s of degree at most $2(N-2)$.

Proof. Clearly we can assume that $k_{n+1}(\sigma)>0$. We let $s_{1}=: s, \operatorname{Re}(s)>2 n, \operatorname{Re}\left(s^{2}\right)>0$ and fix distinct $s_{2}, \ldots, s_{N}$ with $\operatorname{Re}\left(s_{j}\right)>2 n, \operatorname{Re}\left(s_{j}^{2}\right)>0$. Then we multiply both sides of (9.2) by $\prod_{j^{\prime}=2}^{N}\left(s_{j^{\prime}}^{2}-s^{2}\right)$ and apply Proposition 7.1 to the left hand side and equation (9.1) and Theorem 5.1 to the right hand side. The proposition follows immediately from the previous computations.

Now we can complete our proof of the meromorphic continuation of the Selberg zeta function $Z(s, \sigma)$ for every $\sigma \in \hat{M}$ and describe its singularities. We say that a meromorphic function $f: \mathbb{C} \rightarrow \mathbb{C}$ has a singularity of order $k \in \mathbb{Z}$ at $s_{0} \in \mathbb{C}$ if $\lim _{s \rightarrow s_{0}}\left(s-s_{0}\right)^{-k} f(s)$ exists and is not zero.

Theorem 9.2. Let $\sigma \in \hat{M}$. Then the Selberg zeta function $Z(s, \sigma)$ has a meromorphic continuation to $\mathbb{C}$. Its singularities associated to spectral parameters are as follows:

(1) If $\sigma=w_{0} \sigma$ at the points $\pm i \sqrt{\lambda_{k}}$ of order $m_{\mathrm{s}}\left(\lambda_{k}, \sigma\right)$, where $\lambda_{k}$ is a non-zero eigenvalue of $A(\sigma)$ and $m_{\mathrm{s}}\left(\lambda_{k}, \sigma\right)$ is the graded dimension of the corresponding eigenspace. Here for $\operatorname{Re}\left(\lambda_{k}\right)<0$ we take the square root with positive imaginary part.

(2) If $\sigma \neq w_{0} \sigma$ at the points $\pm i \mu_{k}$ of order $\frac{1}{2}\left(m_{\mathrm{s}}\left(\mu_{k}^{2}, \sigma\right)+d\left( \pm \mu_{k}, \sigma\right)-d\left(\mp \mu_{k}, \sigma\right)\right)$. Here $\mu_{k}$ is a non-zero eigenvalue of $D(\sigma)$ of multiplicity $d\left(\mu_{k}, \sigma\right)$ and $m_{\mathrm{s}}\left(\mu_{k}^{2}, \sigma\right)$ is the graded dimension of the eigenspace of $A(\sigma)$ corresponding to the eigenvalue $\mu_{k}^{2}$.

(3) At the point $s=0$ of order $2 m_{\mathrm{s}}(0, \sigma)-c_{1}(\sigma)$ if $\sigma=w_{0} \sigma$ and of order $m_{\mathrm{s}}(0, \sigma)$ if $\sigma \neq w_{0} \sigma$. Here $c_{1}(\sigma)$ is as in (5.9).

(4) At the points $-\beta(\sigma)$ where $\beta(\sigma)$ are the poles of $\operatorname{det}\left(I(\sigma) \circ \mathbf{C}\left(\nu_{\sigma}: \sigma: s\right)\right)$ on $(0, n]$. The order at $\beta(\sigma)$ is $-m(\beta(\sigma))$, where $m(\beta(\sigma))$ is the corresponding multiplicity divided by $\operatorname{dim}\left(\nu_{\sigma}\right)$ as in section 6 .

(5) If $n$ is even at the points $\eta(\sigma)$ of order $m(\eta(\sigma))$ and if $n$ is odd at the points $\eta\left(w_{0} \sigma\right)$ of order $m\left(\eta\left(w_{0} \sigma\right)\right)$. Here $\eta(\sigma)$ resp. $\eta\left(w_{0} \sigma\right)$ are the poles of $\operatorname{det}\left(I(\sigma) \circ \mathbf{C}\left(\nu_{\sigma}: \sigma: s\right)\right)$ resp. $\operatorname{det}\left(I(\sigma) \circ \mathbf{C}\left(\nu_{\sigma}: \sigma: s\right)\right)$ with negative real part and $m(\eta(\sigma)), m\left(\eta\left(w_{0} \sigma\right)\right.$ are the corresponding multiplicities divided by $\operatorname{dim}\left(\nu_{\sigma}\right)$ as in section 6 .

Moreover, the Selberg zeta function $Z(s, \sigma)$ has singularities which depend on $\Gamma$ only via $p$, the number of cusps of $\Gamma$. If all $k_{j}(\sigma)$ are integers, they are located at the negative integers and if all $k_{j}(\sigma)$ are half integers, they are located at the negative half integers. Explicitly, if all $k_{j}(\sigma)$ are integers, they are given as follows.

(1) At the points $-l, l \in \mathbb{N}, l \geq m_{0}$ of order $-p \operatorname{dim}(\sigma)$.

(2) At the points $-l, l \in \mathbb{N}, m_{0} \leq l<\left|k_{j}(\sigma)\right|+\rho_{j}, j=2, \ldots, n+1$ of order $p c_{j, l}(\sigma)$. 
Here $m_{0}$ and the $c_{j, l}(\sigma)$ are as in Proposition 5.4. If all $k_{j}(\sigma)$ are half integers, they can be described in the same way if $\mathbb{N}$ is replaced by $\frac{1}{2} \mathbb{N}-\mathbb{N}$.

The above enumeration exhausts all possible singularities of non-zero order of $Z(s, \sigma)$ if at overlapping singularities the orders are added up.

Proof. Assume that all $k_{j}(\sigma)$ are integers. If $\sigma=w_{0} \sigma$, the Theorem follows from Remark 4.3 and Proposition 7.2. It remains to consider the case that $\sigma \neq w_{0} \sigma$. By Proposition 8.1, the eigenvalues $\lambda_{k}$ of $A(\sigma)$ are given by the $\mu_{k}^{2}$, where $\mu_{k}$ are the eigenvalues of $D(\sigma)$. Moreover one has $Z^{\prime}(s, \sigma) / Z(s, \sigma)=\frac{1}{2}\left(S^{\prime}(s, \sigma) / S(s, \sigma)+S_{a}^{\prime}(s, \sigma) / S_{a}(s, \sigma)\right)$. Thus, combining Proposition 7.2 and Proposition 9.1, it follows that the logarithmic derivative of $Z(s, \sigma)$ has a meromorphic continuation to $\mathbb{C}$ and that its poles with the corresonding residues are as in the enumeration. It remains to show that its residues are integral. For every $k$ let $\mathcal{E}\left( \pm \mu_{k}\right)$ be the eigenspaces of $D(\sigma)$ corresponding to the eigenvalue $\pm \mu_{k}$ and let $\mathcal{E}\left(\mu_{k}^{2}\right)$ be the eigenspace of $A(\sigma)$ corresponding to the eigenvalue $\mu_{k}^{2}$. Then one has $\mathcal{E}\left(\mu_{k}^{2}\right)=\mathcal{E}\left(\mu_{k}\right) \oplus \mathcal{E}\left(-\mu_{k}\right)$. Recall that $m_{\mathrm{s}}\left(\mu_{k}^{2}, \sigma\right)=\operatorname{dim}_{\mathrm{gr}} \mathcal{E}\left(\mu_{k}^{2}\right)$. Thus $m_{\mathrm{s}}\left(\mu_{k}^{2}, \sigma\right)+d\left(\mu_{k}, \sigma\right)-d\left(-\mu_{k}, \sigma\right)$ is even. If all $k_{j}(\sigma)$ are half-integral, one proceeds in the same way by making the appropriate modifications in Proposition 5.4 resp. Proposition 7.2 .

\section{REFERENCES}

[AS] M. Atiyah, W. Schmid, A geometric construction of the discrete series for semisimple Lie groups, Invent. Math. 42 (1977), 1-62

[BON] R. L. Bishop, B. O'Neill, Manifolds of negative curvature, Trans. Amer. Math. Soc. 145 (1969), $1-49$.

[BM] D. Barbasch, H. Moscovici, $L^{2}$-index and the trace formula, J. Funct. An. 53 (1983), no.2, 151-201

[BO] U.Bunke, M. Olbrich, Selberg zeta and theta functions, Akademie Verlag, Berlin (1995)

[BV] N. Bergeron, A. Venkatesh, The asymptotic growth of torsion homology for arithmetic groups, http://arxiv.org/abs/1004.1083 (2010).

[Che] J. Cheeger, Analytic torsion and the heat equation, Ann. of Math. (2) 109 (1979), 259-322

[Do] H. Donnelly, On the cuspidal spectrum for finite volume symmetric spaces, J. Differential Geom. 17 (1982), no.2, 239-253

[EKM] Eguchi, Masaaki; Koizumi, Shin; Mamiuda, Masaichi, The expressions of the Harish-Chandra $C$-functions of semisimple Lie groups $\operatorname{Spin}(n, 1)$, SU(n,1). J. Math. Soc. Japan 51 (1999), no. 4, 955-985.

[Fr] D. Fried, The zeta functions of Ruelle and Selberg I, Ann. Sci. Ecole Norm. Sup. (4) 19 (1986), no. 4, 491-517.

[Ga] R. Gangolli, The length spectra of some compact manifolds of negative curvature, J. Differential Geom. 12 (1977), no. 3, 403-424,

[GaWa] R. Gangolli, G. Warner, Zeta functions of Selberg's type for some non-compact quotients of symmetric spaces of rank one, Nagoya Math. J. 78 (1980), 1-44.

[GP] Y. Gon, J. Park, The zeta functions of Ruelle and Selberg for hyperbolic manifolds with cusps, Math. Ann. 346, no. 3, (2010), 719-767

[GW] R. Goodman, N. Wallach, Representations and invariants of the classical groups, Encyclopedia of Mathematics and its Applications, 68. Cambridge University Press, Cambridge, 1998

[HC] Harish-Chandra, Harmonic analysis on real reductive groups. III. The Maass-Selberg relations and the Plancherel formula. Ann. of Math. (2) 104 (1976), no. 1, 117-201. 
[Ho1] W. Hoffmann, The Fourier transform of weighted orbital integrals on semisimple groups of real rank one, J. Reine Angew. Math. 489 (1997), 53-97

[Ho2] W. Hoffmann, An invariant trace formula for rank one lattices, Math. Nachr. 207 (1999), 93-131.

[Kn] A. Knapp, Representation theory of semisimple groups, Princeton University Press, Princeton, 2001

[KS] A. Knapp, E. Stein, Intertwining operators for semisimple Lie groups, Annals of Math. 93 (1971), 489-578

[LM] H.B. Lawson, M.-L. Michelsohn, Spin geometry, Princeton Mathematical Series, 38. Princeton University Press, Princeton, NJ, 1989.

[MePo] P. Menal-Ferrer, J. Porti, Higher dimensional Reidemeister torsion invariants for cusped hyperbolic 3-manifolds, Preprint 2011, arXiv:1110.3718

[MS] H. Moscovici, R. Stanton, Eta invariants of Dirac operators on locally symmetric manifolds, Invent. Math. 95 (1989), no. 3, 629-666

[Mü1] W. Müller, Analytic torsion and R-torsion of Riemannian manifolds, Adv. in Math. 28 (1978), 233-305

[Mü2] W. Müller, The trace class conjecture in the theory of automorphic forms, Ann. of Math. (2) 130 (1989), no. 3, 473-529

[Mü3] W. Müller, Analytic torsion and R-torsion for unimodular representations, J. Amer. Math. Soc. 6 (1993), 721-753

[MP1] W. Müller, J. Pfaff, The asymptotics of the Ray-Singer analytic torsion for hyperbolic manifolds, Preprint 2011, arXiv:1108.2454.

[MP2] W. Müller, J. Pfaff, Analytic torsion of complete hyperbolic manifolds of finite volume, Preprint 2011, arXiv:1110.4065

[Ter] A. Terras, Harmonic analysis on symmetric spaces and applications I, Springer, New York, 1985.

[OW] M. Osborne, G. Warner, Multiplicities of the Integrable discrete series: the case of a nonuniform lattice in an R-rank one semisimple group, J. Funct. An. 30 (1978), no. 3, 287-310

[Wal] N. Wallach, On the Selberg trace formula in the case of compact quotient, Bull. Amer. Math. Soc. 82 (1976), no.2, 171-195

[War] G. Warner, Selberg's trace formula for nonuniform lattices: the R-rank one case, Studies in algebra and number theory, Adv. in Math. Suppl. Stud. 6, Academic Press, New York-London, 1979

[Wo] A. Wotzke, Die Ruellesche Zetafunktion und die analytische Torsion hyperbolischer Mannigfaltigkeiten, Bonner Mathematische Schriften 389 (2008)

Universität Bonn, Mathematisches Institut, Endenicher Alle 60, D - 53115 Bonn, GerMANY

E-mail address: pfaff@math.uni-bonn.de 